\title{
Endothelial-to-mesenchymal transition drives atherosclerosis progression
}

\author{
Pei-Yu Chen, ${ }^{1}$ Lingfeng Qin, ${ }^{2}$ Nicolas Baeyens, ${ }^{1}$ Guangxin Li, ${ }^{2}$ Titilayo Afolabi, ${ }^{1}$ Madhusudhan Budatha, ${ }^{1}$ George Tellides, ${ }^{2}$ \\ Martin A. Schwartz, ${ }^{1,3,4}$ and Michael Simons ${ }^{1,3}$ \\ 'Yale Cardiovascular Research Center, Department of Internal Medicine, ${ }^{2}$ Department of Surgery, ${ }^{3}$ Department of Cell Biology, and ${ }^{4}$ Department of Biomedical Engineering, \\ Yale University School of Medicine, New Haven, Connecticut, USA.
}

\begin{abstract}
The molecular mechanisms responsible for the development and progression of atherosclerotic lesions have not been fully established. Here, we investigated the role played by endothelial-to-mesenchymal transition (EndMT) and its key regulator FGF receptor 1 (FGFR1) in atherosclerosis. In cultured human endothelial cells, both inflammatory cytokines and oscillatory shear stress reduced endothelial FGFR1 expression and activated TGF- $\beta$ signaling. We further explored the link between disrupted FGF endothelial signaling and progression of atherosclerosis by introducing endothelial-specific deletion of FGF receptor substrate $2 \alpha$ (Frs2a) in atherosclerotic $\left(\mathrm{Apo}^{-\mathrm{C}^{-}}\right)$mice. When placed on a high-fat diet, these double-knockout mice developed atherosclerosis at a much earlier time point compared with that their $\mathrm{Apoe}^{-/-}$counterparts, eventually demonstrating an $\mathbf{8 4} \%$ increase in total plaque burden. Moreover, these animals exhibited extensive development of EndMT, deposition of fibronectin, and increased neointima formation. Additionally, we conducted a molecular and morphometric examination of left main coronary arteries from $\mathbf{4 3}$ patients with various levels of coronary disease to assess the clinical relevance of these findings. The extent of coronary atherosclerosis in this patient set strongly correlated with loss of endothelial FGFR1 expression, activation of endothelial TCF- $\beta$ signaling, and the extent of EndMT. These data demonstrate a link between loss of protective endothelial FGFR signaling, development of EndMT, and progression of atherosclerosis.
\end{abstract}

\section{Introduction}

Atherosclerosis is a progressive disorder initiated by biomechanical forces in areas of the vascular tree subjected to disturbed blood flow and number of systemic factors, including hyperlipidemia, smoking, and diabetes $(1,2)$. Lipid uptake by the vascular wall leads over time to a gradual buildup of atherosclerotic plaques. Once formed, the plaque continues to expand, leading to a gradual restriction in lumen diameter and compromise of blood flow. Under certain conditions, plaque rupture can precipitate intravascular thrombosis, resulting in complete and sudden interruption of the arterial blood supply. The buildup, growth, and rupture of the plaque have all been associated with the presence of systemic and vascular wall inflammation (3-5). Despite strong data linking vessel wall inflammation to atherosclerosis progression, the mechanism(s) of inflammation-induced atherosclerotic disease progression remains obscure (4), while efforts to halt disease progression by antiinflammatory therapies have failed in clinical trial (3).

Recent studies have documented a high incidence of endothelial-to-mesenchymal transition (EndMT) in a number of pathological conditions associated with inflammation, such as myocardial infarction (6), cerebral cavernous malformations (7), portal hypertension (8), pulmonary hypertension (9), and vascular graft failure $(10,11)$ among others. EndMT is characterized by a change in phenotype of normal endothelial cells (ECs) that assume the shape

Authorship note: Pei-Yu Chen and Lingfeng Qin contributed equally to this work. Conflict of interest: The authors have declared that no conflict of interest exists. Submitted: May 7, 2015; Accepted: September 17, 2015.

Reference information: J Clin Invest. 2015;125(12):4514-4528. doi:10.1172/JCI82719. and properties of mesenchymal cells (fibroblasts, smooth muscle cells [SMCs]), including enhanced proliferation and migration; secretion of extracellular matrix proteins, such as fibronectin and collagen; and expression of various leukocyte adhesion molecules.

TGF- $\beta$ has been identified as the central player in driving EndMT progression $(12,13)$, but the processes leading to activation of its signaling remain poorly defined. We have previously reported that a reduction in endothelial FGF-mediated signaling induced by knockout of either the key intracellular signal mediator FGF receptor substrate $2 \alpha(\mathrm{FRS} 2 \alpha)$ (10) or the primary FGF receptor in the endothelium (FGF receptor 1 [FGFR1]) (14) activates TGF- $\beta$ signaling, leading to EndMT.

The unusual feature of FGFR1 biology is that FGFR1's expression in ECs is affected by certain inflammatory stimuli, including IFN- $\gamma$, TNF- $\alpha$, and IL-1 $\beta$, that induce a profound reduction in its expression (10). Since these are precisely the inflammatory mediators found in atherosclerotic plaques, we set out to investigate the occurrence and role of EndMT in atherosclerosis.

Studies in cell culture and mouse models demonstrated that both oscillatory fluid shear stress and soluble inflammatory mediators reduced FGFR1 expression and signaling and promoted TGF- $\beta$ signaling and EndMT. In addition, analysis of human coronary arteries demonstrated a strong correlation among endothelial FGFR1 expression, increased TGF- $\beta$ signaling, and the appearance of EndMT with the severity of atherosclerosis. Together, these findings point to a mechanism whereby biomechanical and soluble inflammatory stimuli reduce protective endothelial FGFR1 signaling to drive EndMT and progression of atherosclerotic plaque. 
A

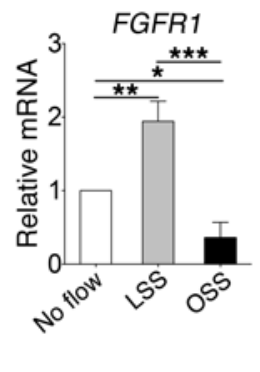

EndMT transcription factor

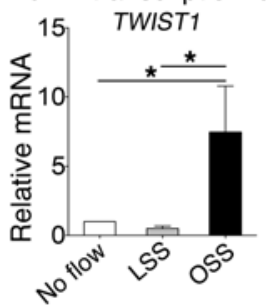

B

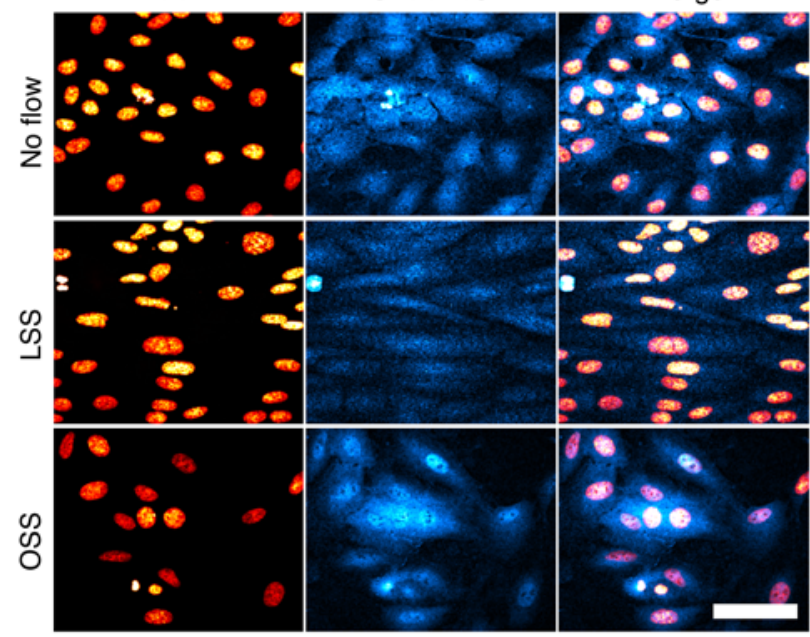

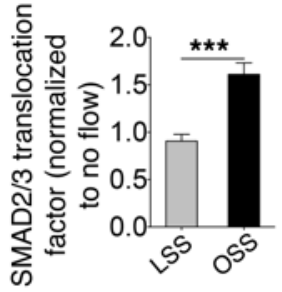

$\mathbf{E}$

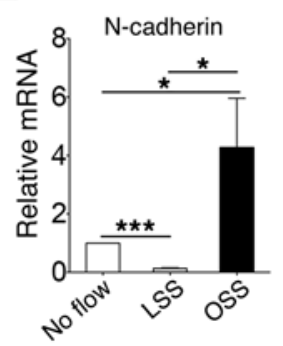

Mesenchymal markers

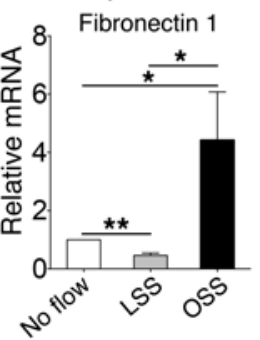

Figure 1. Shear stress downregulates FGFR1 expression and upregulates TGF- $\beta$ signaling in vitro. (A and B) HUVECs were subject to 12 dynes/cm ${ }^{2}$ LSS or $1 \pm 4$ dynes $/ \mathrm{cm}^{2}$ OSS for 16 hours. FGFR 1 expression and SMAD2/3 nuclear translocation were examined by (A) qRT-PCR and (B) immunofluorescence staining. The bar graph of qRT-PCR results is representative of 4 independent experiments $\left({ }^{*} P<0.05 ;{ }^{* *} P<0.01\right.$; ${ }^{* * *} P<0.001 ; 1$-way ANOVA with NewmanKeuls post-hoc test for multiple comparison correction). SMAD2/3 nuclear translocation images are representative of 3 independent experiments ( ${ }^{* *} P<0.001$; unpaired 2-tailed Student's $t$ test). Scale bar: $50 \mu \mathrm{m}$. (C-E) HUVECs were subject to 12 dynes/cm² LSS or $1 \pm 4$ dynes/cm² $0 S S$ for 48 hours. EndMT marker gene expression was examined by qRT-PCR. Bar graphs of qRT-PCR results are representative of 4 independent experiments $\left({ }^{*} P<0.05\right.$; ${ }^{*} P<0.01$; 1-way ANOVA with Newman-Keuls post-hoc test for multiple comparison correction).

\section{Results}

Inflammatory cytokines, shear stress, and EndMT. Inflammation and shear stress play important roles in the development and progression of atherosclerotic lesions. Previous studies have identified FGFR1 expression as a key regulator of TGF- $\beta$-induced EndMT (14) and demonstrated that certain inflammatory cytokines could reduce FGFR1 levels, thereby reducing FGF signaling (10). To test the link between the extent of reduction in FGF signaling, FGFR1 expression, and cytokines, we first studied the relationship between the reduction in FGFR1, or its key intracellular signaling mediator FRS $2 \alpha$ expression, and activation of TGF- $\beta$ signaling and the appearance of EndMT. When exposed to progressively higher doses of FGFR1 or FRS2 $\alpha$ shRNA-encoding lentiviruses, HUVECs exhibited a dose-dependent decline in target gene expression, an increase in phosphorylation of TGF- $\beta$ signaling intermediary SMAD2 (p-SMAD2), and the appearance of EndMT markers collagen 1, vimentin, and SM22 $\alpha$ (Supplemental Figure 1; see complete unedited blots in the supplemental material; supplemental material available online with this article; doi:10.1172/JCI82719DS1).

HUVECs were next treated with increasing concentrations of IFN- $\gamma$, TNF- $\alpha$, or IL- $1 \beta$. All 3 cytokines induced a decrease in
FGFR1 expression and an increase in EndMT markers (Supplemental Figure 2; see complete unedited blots in the supplemental material) that was similar to results observed with shRNA-mediated suppression of FGF signaling described above. To more fully characterize this response, HUVECs were treated with optimal doses of the 3 cytokines, alone or in combination. The cytokineinduced reduction in FGFR1 expression was most pronounced when IFN- $\gamma$, TNF- $\alpha$, and IL-1 $\beta$ were used together (Supplemental Figure 3B; see complete unedited blots in the supplemental material). On their own, none of the cytokines induced a reduction in expression of endothelial markers, such as vascular endothelial-cadherin (VE-cadherin) or VEGFR2. However, cytokine combinations profoundly reduced expression of these proteins (Supplemental Figure 3B; see complete unedited blots in the supplemental material). Similarly, an increase in TGF- $\beta$ receptor 1 (TGF$\beta R 1)$ expression, a key step in EndMT induction, was most pronounced when any 2 of the 3 cytokines were present (Supplemental Figure 3A). Finally, induction of EndMT in ECs in vitro resulted in a marked upregulation of mesenchymal markers (ZEB2, SLUG, SNAIL, vimentin, and collagen 1 ), smooth muscle marker (SM22 $\alpha$ ), leukocyte adhesion molecules (ICAM-1 and VCAM-1), monocyte 
A

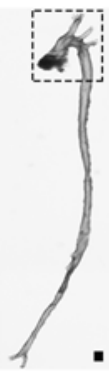

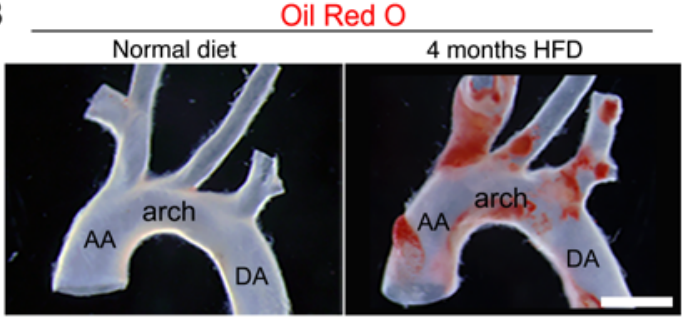

C

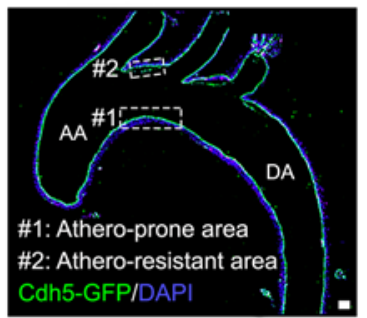

D

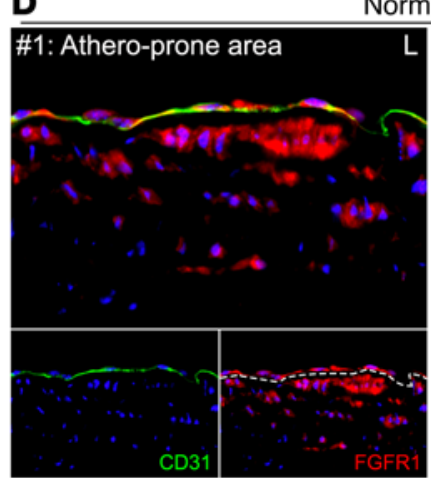

Normal diet

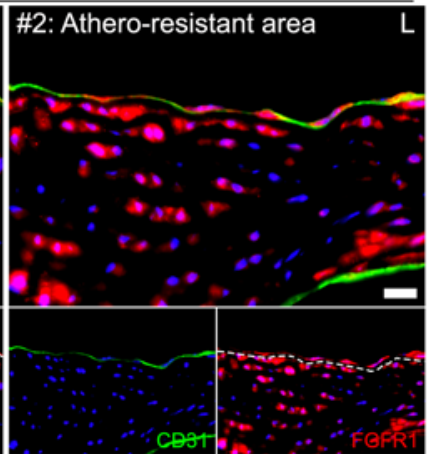

CD31/FGFR1/DAPI

$\mathbf{F}$

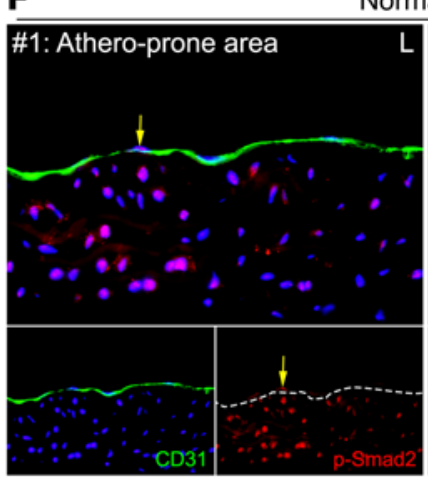

Normal diet

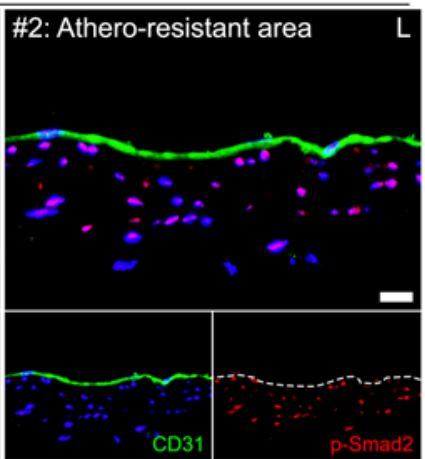

CD31/p-SMAD2/DAPI
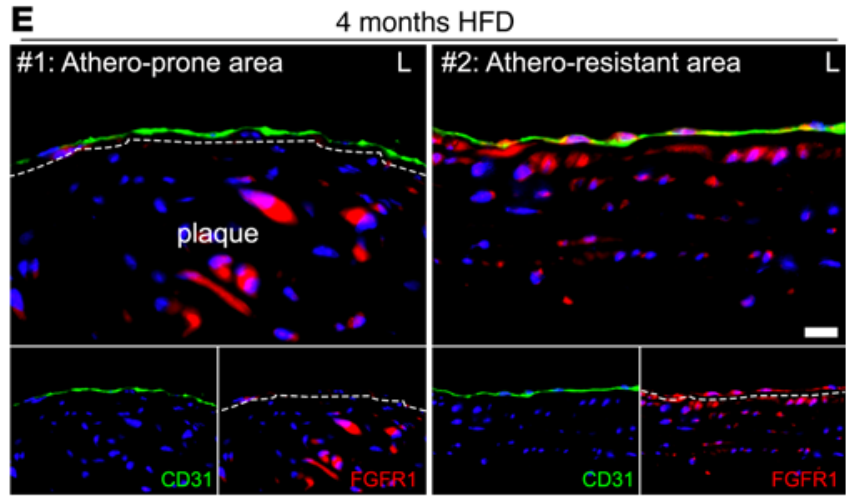

CD31/FGFR1/DAPI

\section{G}

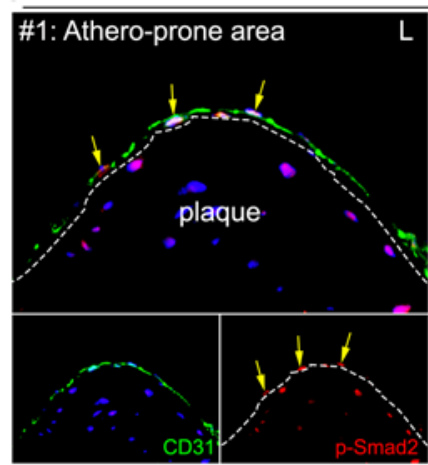

4 months HFD

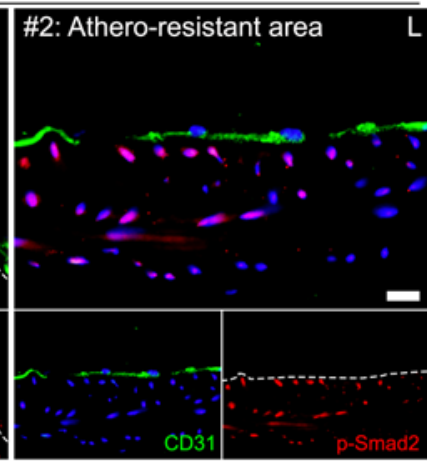

CD31/p-SMAD2/DAPI
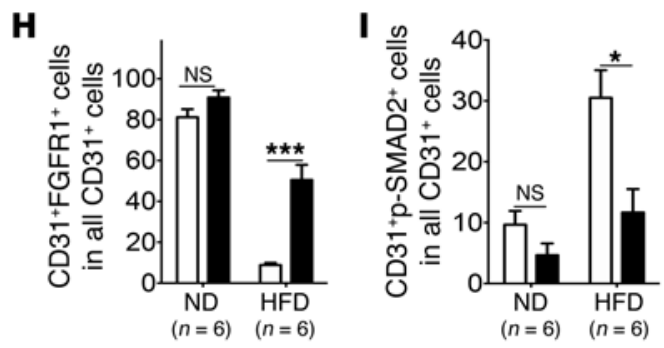

Figure 2. Shear stress downregulates FGFR1 expression and upregulates TGF- $\boldsymbol{\beta}$ signaling in vivo. (A) Whole mouse aorta. Scale bar: 1 mm. (B) Representative photomicrographs of Oil Red O-stained aortic arch sections of Apoe ${ }^{-/-}$mice after 16 weeks on the normal diet $(n=3)$ or HFD ( $n=3$ ). Scale bar: $4 \mathrm{~mm}$. (C) A high-magnification image of $\mathbf{A}$, showing the aortic arch in the longitudinal section. AA, ascending aorta; DA, descending aorta. Scale

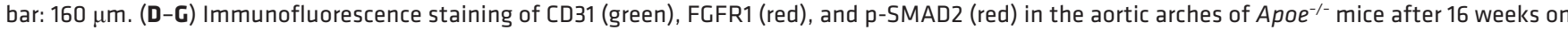
normal diet $(n=6)$ or HFD $(n=6)$. Atherosclerosis-prone areas are denoted by \#1, while an atherosclerosis-resistant areas are denoted by \#2. Yellow arrows indicate ECs expressing P-SMAD2. Nuclei were stained with DAPI (blue). L, lumen. Scale bar: $10 \mu \mathrm{m}$. (H and I) Quantification of the number of luminal EC expressing FGFR1 and p-SMAD2 (NS, not significant compared with \#1; ${ }^{*}<0.05$ compared with \#1; *** $P<0.001$ compared with \#1; unpaired 2-tailed Student's $t$ test). 


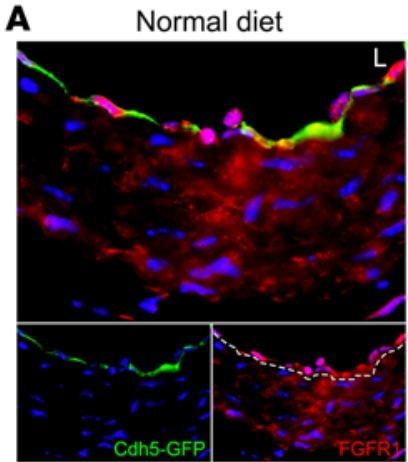

C

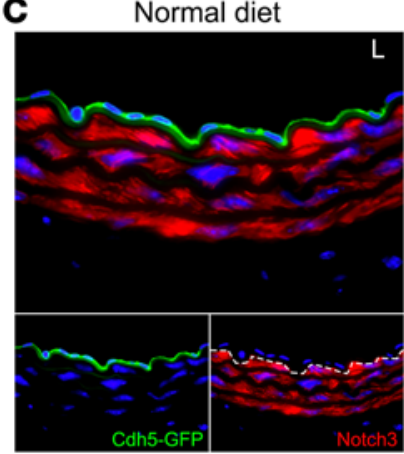

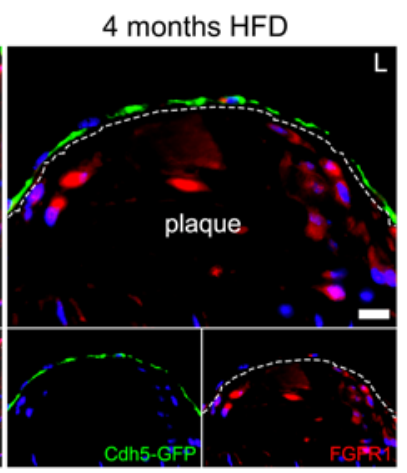

Cdh5-GFP/FGFR1/DAPI

4 months HFD

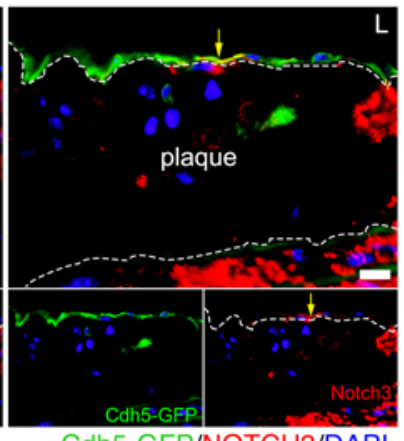

B

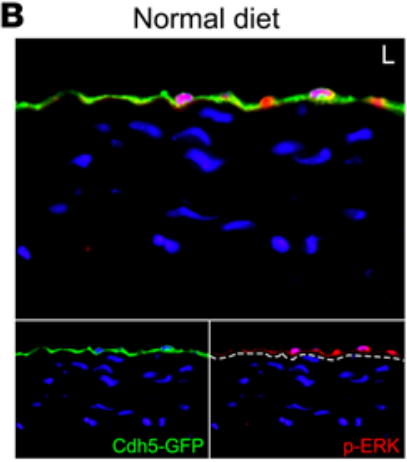

D

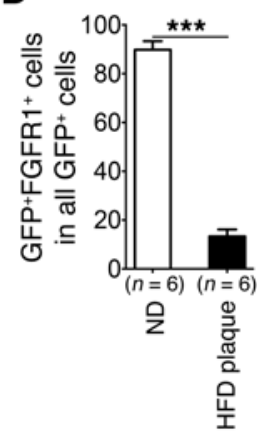

E

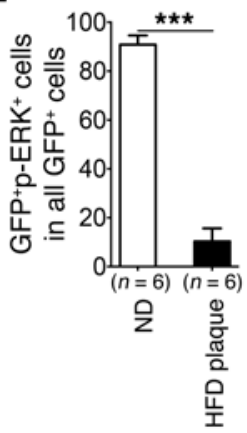

4 months HFD

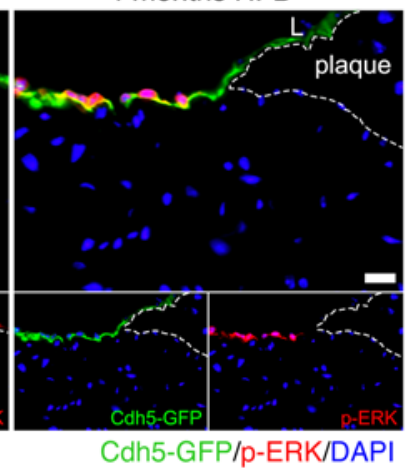

$\mathbf{F}$

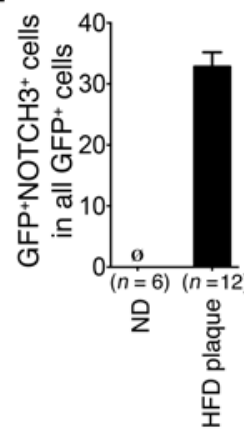

Figure 3. FGF signaling activity and EndMT extent in a mouse atherosclerosis model. (A) Immunofluorescence staining of FGFR1 in aortas of Cdh5CreER ${ }^{T 2} \mathrm{mT} / \mathrm{mG}$ Apoe ${ }^{-/-}$mice (6 mice per group). Scale bar: $10 \mu \mathrm{m}$. (B) Cdh5-CreER ${ }^{T 2} \mathrm{mT} / \mathrm{mG}$ Apoe (- $^{-1}$ mice after 16 weeks on HFD ( $\left.n=6\right)$ or normal diet $(n=6)$ were injected i.v. with FGF2 ( $1 \mu \mathrm{g}$ per mouse), and p-ERK activation was determined by immunofluorescence staining. Scale bar: $10 \mu \mathrm{m}$. (C) Immunofluorescence staining of NOTCH3 in aorta and plaque sections in Cdh5-CreER ${ }^{T 2} \mathrm{mT} / \mathrm{mG}$ Apoe ${ }^{-/-}$mice (normal diet, $n=6 ; \mathrm{HFD}, n=12$ ). Scale bar: $10 \mu \mathrm{m}$. (D-F) Quantification of the number of luminal ECs expressing FGFR1, p-ERK, and NOTCH3 ( ${ }^{* * *} P<0.001$ compared with normal diet; unpaired 2-tailed Student's $t$ test). Yellow arrows indicate ECs expressing NOTCH3. Ø, not detected.

chemotactic protein 1 (MCP-1), and proinflammatory protein plasminogen activator inhibitor-1 (PAI-1) and the loss of protective protein endothelial NOS (eNOS) (Supplemental Figure 3B; see complete unedited blots in the supplemental material). Interestingly, IFN- $\gamma$, TNF- $\alpha$, or IL-1 $\beta$ treatments did not affect FGFR1 expression in primary human SMCs (Supplemental Figure 4, B and D; see complete unedited blots in the supplemental material).

Atherosclerotic plaques preferentially develop in regions of arteries characterized by oscillating shear stress (OSS), in contrast to high laminar shear stress (LSS), which is atheroprotective. We therefore examined the effect of OSS and LSS on FGFR1 expression and TGF- $\beta$ signaling in HUVECs. When exposed to 16 hours of OSS, HUVECs demonstrated a significant reduction in FGFR1 expression, while a similar duration of exposure to LSS increased it (Figure 1A). Staining for SMAD2/3 demonstrated increased translocation of these proteins to nuclei of HUVECs exposed to OSS, but not LSS, a finding consistent with activation of TGF- $\beta$ signaling by OSS (Figure 1B). In addition, OSS, but not LSS, induced a significant increase in mRNA levels of the transcription factor TWIST1 (Figure 1C), which has been reported to be involved with TGF- $\beta$ signaling $(11,15)$. In agreement with this activation of the EndMT program, there was an increase in expression of SMC markers (ACTA2, which encodes smooth muscle $\alpha$-actin, and NOTCH3) (Figure 1D) and mesenchymal markers (N-cadherin, fibronectin 1 , and collagen $1 \mathrm{~A}$ ) (Figure 1E).
Disturbed shear stress is present in certain parts of the arterial vascular tree, such as the lesser curvature of the aortic arch and branch origin sites, while an atheroprotective linear shear stress is present in other sites, including the greater curvature of the arch (Figure 2, A-C). Indeed, after 4 months of high-fat diet (HFD), Apoe $^{-/-}$mice had extensive lesions in atheroprone areas but not in atheroprotected areas (Figure 2B). Immunocytochemical examination of aorta sections from mice on normal diet did not show any difference in endothelial FGFR1 expression (Figure 2, D and $\mathrm{H}$ ) or p-SMAD2 levels (Figure 2, F and I) between atheroprone and atheroprotected areas. However, after 4 months of HFD, FGFR1 expression was significantly lower, and p-SMAD2 levels were significantly higher, in the atheroprone endothelium compared with the atheroprotected endothelium (Figure 2, E and G-I).

EndMT in mouse atherosclerosis. To establish a causal relationship between the loss of endothelial FGF signaling and atherosclerosis, we next examined the impact of HFD on ECs in endothelial fate-mapped $m T / m G$ reporter Apoe $/-$ mice (Cdhr5-CreER ${ }^{T 2} \mathrm{mT}$ / $m G$ Apoe $^{-/}$mice). Sixteen weeks of HFD resulted in a profound reduction of FGFR1 expression in luminal ECs (Figure 3, A and D). To examine the functional impact of this reduction in FGFR1 levels, mice on normal diet and HFD were injected systemically with FGF2. Immunocytochemical analysis of p-ERK expression in the aorta 15 minutes after FGF2 injection demonstrated a dramatic 
A

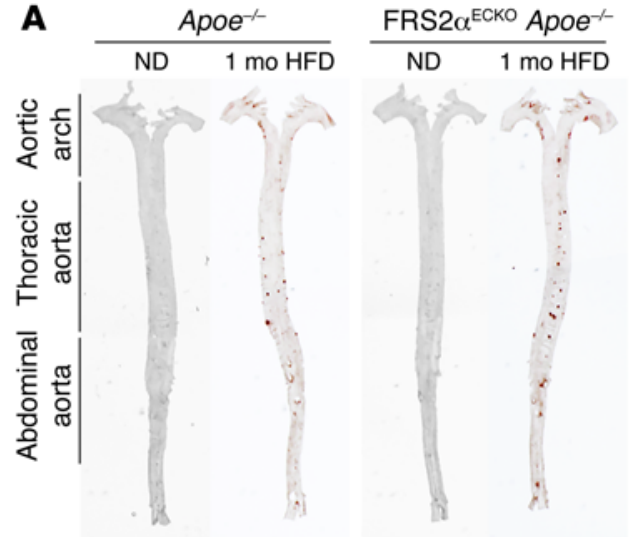

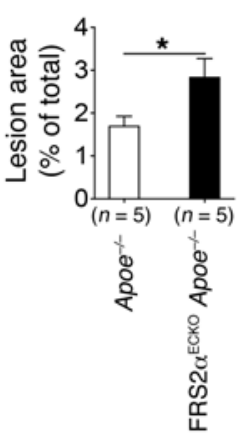

B

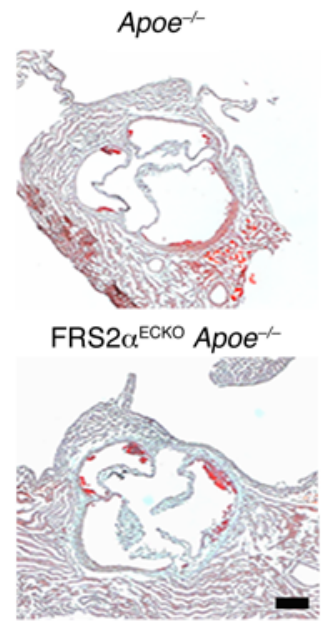

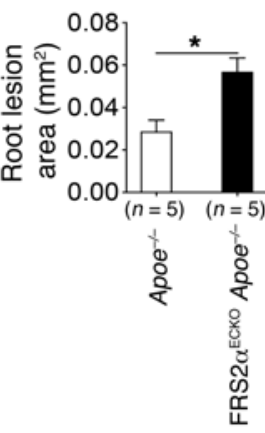

Figure 4. Effect of endothelial FGF signaling suppression on atherosclerosis in mice after 4 weeks of $\mathbf{H F D}$. Apoe $e^{-/-}$and FRS2 $\alpha^{\mathrm{ECKO}} A p o e^{-/-}$mice were placed on a HFD and examined 4 weeks later. (A) Microphotographs of aortas from $A p o e^{-/-}$and FRS2 $\alpha^{\mathrm{ECKO}} A p o e^{-/-}$mice at 12 weeks of age in the en face preparation after staining with Oil Red 0 ( 5 mice per group) and quantification of lesion area. All data are shown as mean $\pm \mathrm{SD}\left({ }^{*} P<0.05\right.$ compared with $A p o e^{-1-}$; unpaired 2-tailed Student's $t$ test). (B) Representative examples of cross sections from the Oil Red 0-stained aortic root (5 mice per group) and quantification of aortic root lesion areas. Scale bar: $200 \mu \mathrm{m}$. Mean \pm SD. $\left({ }^{*} P<0.05\right.$ compared with $A p o e^{-l-}$; unpaired 2-tailed Student's $t$ test).

reduction of p-ERK in plaque compared with that in normal ECs from vessel segments in mice on normal diet (Figure 3, B and E). In agreement with this reduction in endothelial FGF signaling, over a third of the luminal ECs in mice on HFD expressed the EndMT marker NOTCH3, which was virtually absent in mice maintained on the normal diet (Figure 3, C and F).

To further examine the role of endothelial FGF signaling in atherosclerotic lesion development, we generated mice with inducible endothelium-specific deletion of the gene encoding the pan-FGFR adaptor molecule FRS2 $\alpha$ on an endothelial fate-mapped Apoe ${ }^{-/-}$ background using a Cdh5-CreER ${ }^{T 2}$ driver (16). Cdh5-CreER ${ }^{T 2} \mathrm{mT} /$ $m G$ mice were crossed with Frs $2 a^{f / f l}$ mice, generating the Cdh5CreER ${ }^{T 2} m T / m G$ Frs $2 a^{f / / l}$ line (hereby designated as FRS2 $\alpha^{\text {ЕСКо }}$ mice). These mice were then crossed on to the Apoe ${ }^{-/}$background, generating Cdh5-CreER $R^{T 2} \mathrm{mT} / \mathrm{mG}$ Apoe ${ }^{-/} \mathrm{Frs} 2 a^{\mathrm{A} / \mathrm{fl}}$ mice (FRS2 $\alpha^{\mathrm{ECKO}}$ Apoe $e^{-/}$mice) and Cdh5-CreER ${ }^{T 2} \mathrm{mT} / \mathrm{mG} \mathrm{Apoe^{-/ }} \mathrm{Frs}^{2 a^{7 / /} \text { littermate }}$ controls (Supplemental Figure 5A). Tamoxifen was injected postnatally, and the efficiency of fate mapping and FRS2 $\alpha$ excision in FRS2 $\alpha^{\text {ECKO }}$ Apoe $^{-/-}$mice was determined by immunocytochemical examination of aortas (Supplemental Figure 5B) and Western blotting (Supplemental Figure 5C; see complete unedited blots in the supplemental material). Body weight, total cholesterol, and triglyceride and HDL cholesterol levels in FRS2 ${ }^{\mathrm{ECKO}} \mathrm{Apoe}^{-/}$mice were no different from those in Apoe ${ }^{-/}$mice before and after 16 weeks of HFD (Supplemental Figure 5, D and E).

Examination of the aortic arch, thoracic and abdominal aorta, and aortic root after 4 weeks of HFD revealed more extensive atherosclerotic lesions in FRS2 ${ }^{\mathrm{ECKO}}$ Apoe ${ }^{-/-}$mice compared with $A p o e^{-/-}$mice (Figure 4). In addition, FRS2 $\alpha^{\mathrm{ECKO}} \mathrm{Apoe}^{-/-}$aortas showed increased atherosclerotic plaque lesions, deposition of fibronectin, and elevated expression of ICAM- 1 and VCAM- 1 in both atherosclerosis-prone and -resistant areas that were not seen in $\mathrm{Apoe}^{-/}$mice (Figure 5).

After 16 weeks of HFD, FRS2 $\alpha^{\text {ЕСKO }}$ Apoe $^{-/-}$mice developed extensive atherosclerotic plaques, involving the aortic arch, thoracic and abdominal aorta, aortic root, and brachiocephalic arteries (Figure 6, A-C). The total plaque burden in the aorta was approximately $84 \%$ larger than that in $A p o e^{-/-}$mice $(17.82 \%$ in $\mathrm{Apoe}^{-/-}$vs. $32.79 \%$ in FRS2 $\alpha^{\mathrm{ECKO}}$ Apoe $^{-/}$) (Figure 6A). Increases in the lesion area were equally significant in the aortic root, brachiocephalic artery, and abdominal aorta (Figure 6, B, C, and E). Histologic examination of the abdominal aorta demonstrated very extensive circumferential plaque in double-knockout animals that covered normally atheroresistant areas that was never observed in $\mathrm{Apoe}^{-/}$mice (Figure 6D). Furthermore, there was a significant increase in the size of the necrotic core and a decrease in the size of the fibrous cap (Figure 6, D and F), features typical of the vulnerable plaque.

Immunocytochemistry demonstrated extensive circumferential expression of ICAM- 1 and VCAM- 1 and the recruitment of monocytes/macrophages (Figure 7, A, B, D, and E). In addition, atherosclerotic lesions of $\mathrm{FRS} 2 \alpha^{\mathrm{ECKO}} \mathrm{Apoe}^{-/-}$mice stained strongly for activated TGF- $\beta$ signaling (p-SMAD2), smooth muscle $\alpha$-actin, and NOTCH3 expression and extensive collagen and fibronectin deposition (Supplemental Figure 6 and Figure 7, C and F), all markers for EndMT. Examination of brachiocephalic arteries produced similar findings (Supplemental Figure 7).

EndMT in human atherosclerosis. To investigate EndMT in human atherosclerosis, immunofluorescence microscopy was used to examine the left main coronary arteries (Supplemental Figure $8 \mathrm{~A}$ ) of patients with normal coronaries (defined as having no neointima), mild coronary artery disease (CAD) (intima-tomedia $[\mathrm{I} / \mathrm{M}]$ ratio, $<0.2$, grade I plaque), moderate $\mathrm{CAD}(\mathrm{I} / \mathrm{M}$ ratio, 0.2-1.0, grade II plaque), or severe CAD (I/M ratio, $>1.0$, grade III and IV plaque) (Supplemental Figure 8B).

While $81 \%$ of the luminal endothelium in patients with no/ mild CAD demonstrated expression of FGFR1, this was reduced to $47.6 \%$ of the luminal endothelium in patients with moderate $\mathrm{CAD}$ and $11.1 \%$ in patients with severe CAD (Figure $8, A$ and B). In the same samples, p-SMAD2, a marker of activated TGF- $\beta$ signaling, 
A

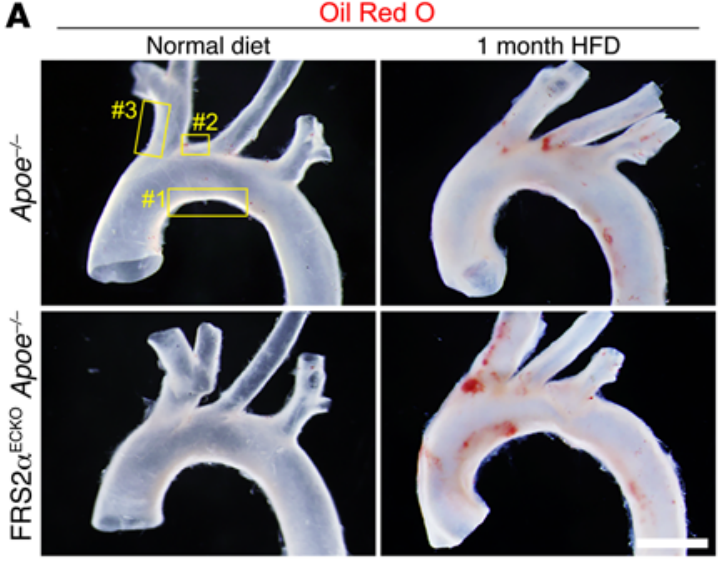

c
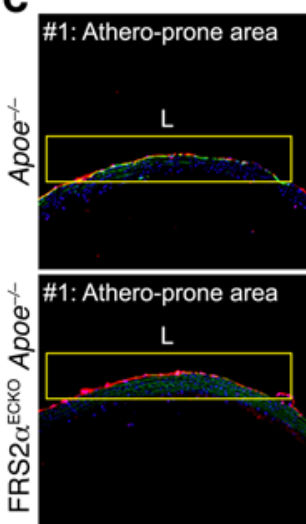
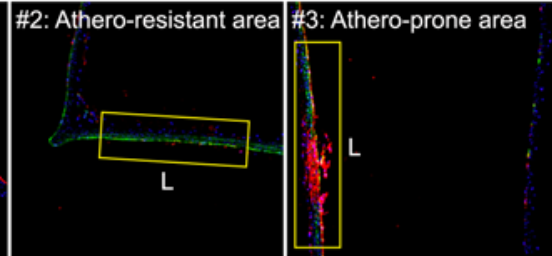

\#2: Athero-resistant area \#3: Athero-prone area

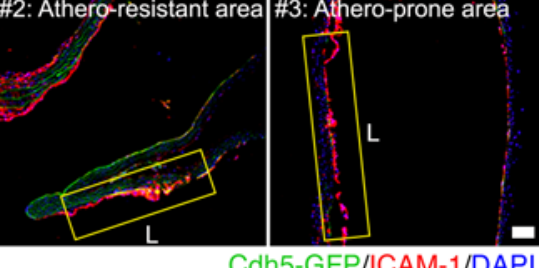

B
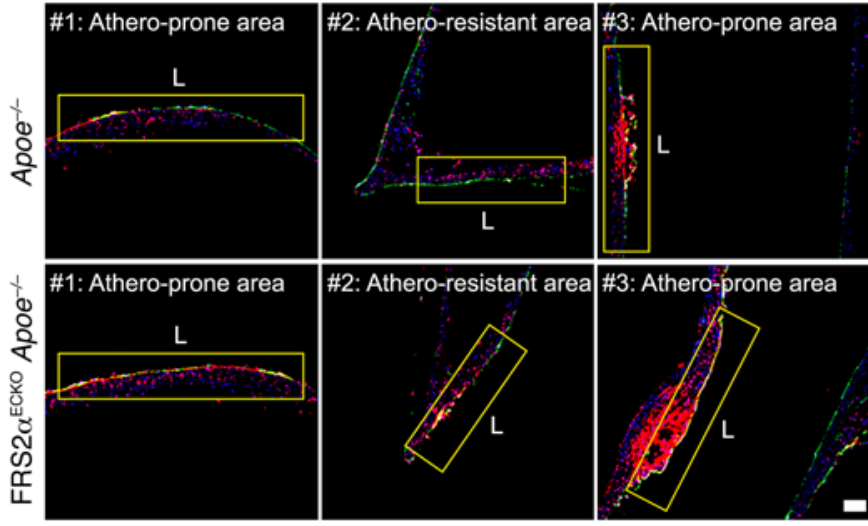

\#2: Athero-resistant area \#3: Athero-prone area

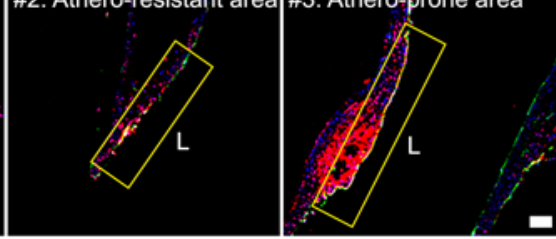

Cdh5-GFP/FN/DAPI

D
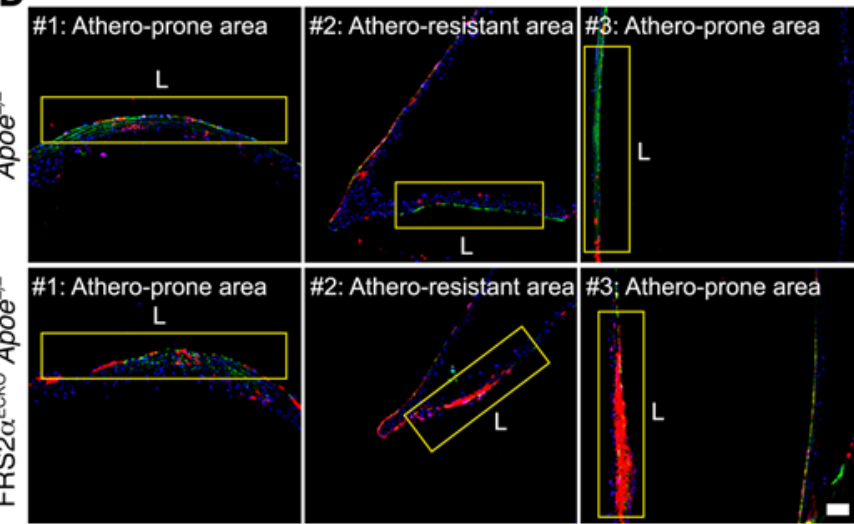

\#2: Athero-resistant area \#3; Athero-prone area

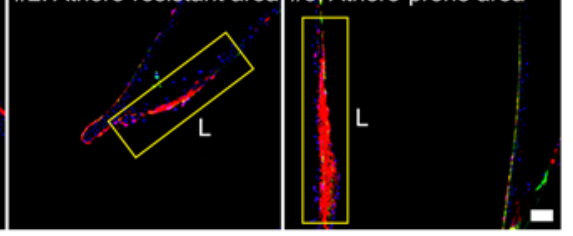

Cdh5-GFP/VCAM-1/DAPI

Figure 5. Early onset of atherogenesis in FRS2 $\alpha^{\mathrm{ECKO}} \mathbf{A p o e}^{-/-}$mice. Apoe ${ }^{-/-}$and FRS2 $\alpha^{\mathrm{ECKO}} \mathrm{Apoe}^{-/-}$mice were placed on a HFD and examined 4 weeks later. (A) Representative photomicrographs of Oil Red O-stained atherosclerotic lesions in the aortic arch before and after 4 weeks of HFD (5 mice per group). \#1 and \#3 denote atherosclerosis-prone areas, while \#2 denotes an atherosclerosis-resistant area. Scale bar: 4 mm. (B-D) Fibronectin (FN), ICAM-1, and VCAM-1 longitudinal sections of areas corresponding to boxes \#1-\#3 from $\mathbf{A}$ in $A p o e^{-/-}$and FRS2 $\alpha^{\text {ЕटКo }}$ Apoe $^{-/-}$mice (5 mice per group). Nuclei were stained with DAPI (blue). Scale bar: $16 \mu \mathrm{m}$.

progressively increased with disease severity (Figure 8, C and D). There was a strong inverse linear relationship between the I/M ratio and number of ECs expressing FGFR1 $(r=-0.84, P<0.0001$ Figure $8 \mathrm{E}$ ) and an equally strong positive correlation with the number of ECs expressing p-SMAD2 $(r=0.83, P<0.0001$, Figure $8 \mathrm{~F})$. Finally, FGFR1 expression was inversely correlated with TGF- $\beta$ signaling $(r=-0.78, P<0.0001$, Figure $8 G)$.

We next examined the EndMT markers NOTCH3 and SM22 $\alpha$ in this patient cohort. While essentially no luminal coronary ECs expressed these proteins in the absence of disease, their prevalence increased significantly with disease severity, with virtually all ECs in the left main arteries from patients with severe CAD showing strong staining (Figure 9, A-D). Quantitative analysis of the entire patient cohort demonstrated a strong correlation between the number of ECs expressing NOTCH3 and SM22 $\alpha$ and the I/M ratio once the prevalence of transformed ECs approached approximately $35 \%(r=0.88$ and 0.75 , respectively; $P<0.0001$, Figure $9, \mathrm{E}$ and $\mathrm{F}$ ).

The extracellular matrix proteins collagen and fibronectin are well-recognized markers of epithelial-to-mesenchymal transition and EndMT and play important roles in atherosclerotic plaque progression. Their expression by ECs in this patient population also strongly correlated with increasing I/M ratio (Figure 10, A-D), and there was a strong linear relationship between increasing endothelial expression of collagen and fibronectin and disease severity (Figure 10, E and F). In addition, immunostaining demonstrated increasing luminal endothelial expression of ICAM-1 and VCAM-1, with increasing CAD severity (Figure 11, A-D). There was a strong correlation between increase in fibronectin deposition and ICAM-1 and VCAM-1 expression (Figure 11, E and F). These observations establish an association between the severity of CAD and loss of endothelial FGFR1 expression as well as between activation of endothelial TGF- $\beta$ signaling and the extent of EndMT.

\section{Discussion}

The results of this study suggest that EndMT serves as the link between factors initiating atherosclerosis, such as inflammation and disturbed shear and the tissue remodeling that leads to plaque formation. The initiating event is the loss of protective endothelial FGF signaling input induced by OSS and vessel wall inflammation. This results in activation of endothelial TGF- $\beta$ signaling and induction of EndMT. Once induced, EndMT promotes further 


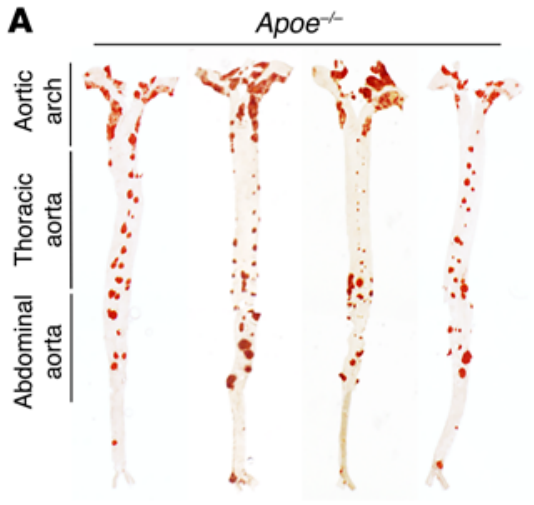

C

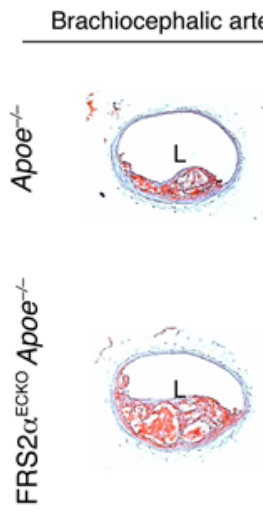

Oil Red O
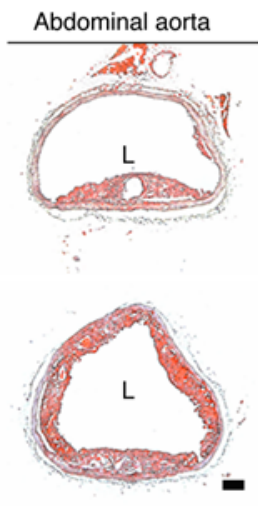

$\mathrm{FRS} 2 \alpha^{\mathrm{ECKO}} \mathrm{Apoe}^{-/-}$

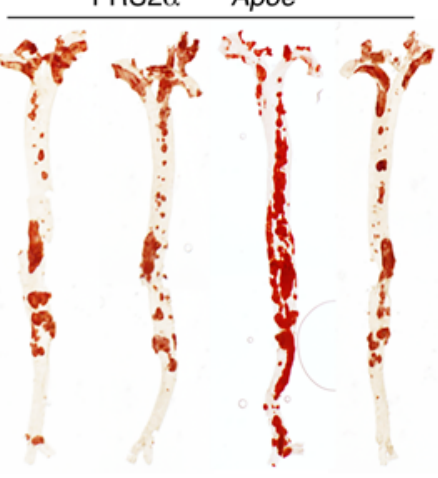

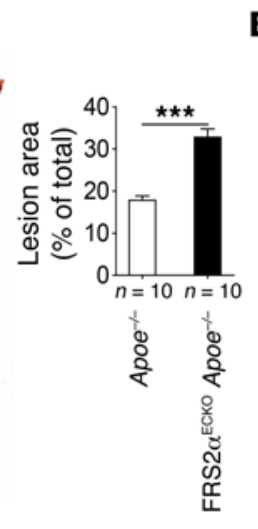

B $\quad \mathrm{Apoe}^{-1-}$

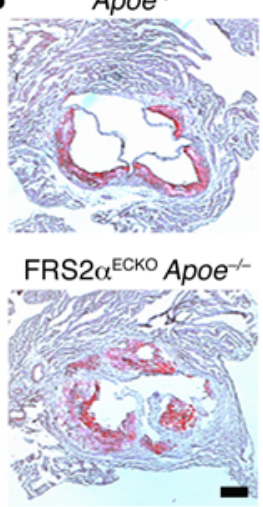

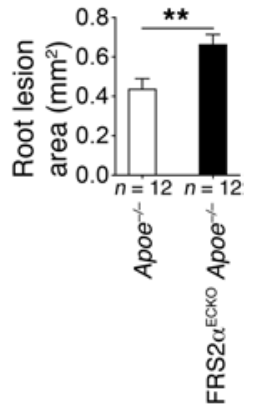

D

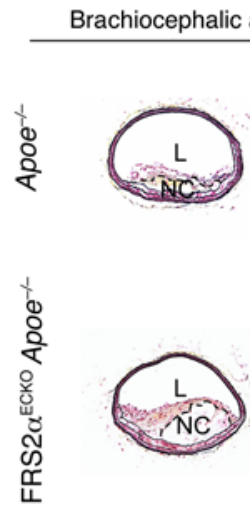

Movat
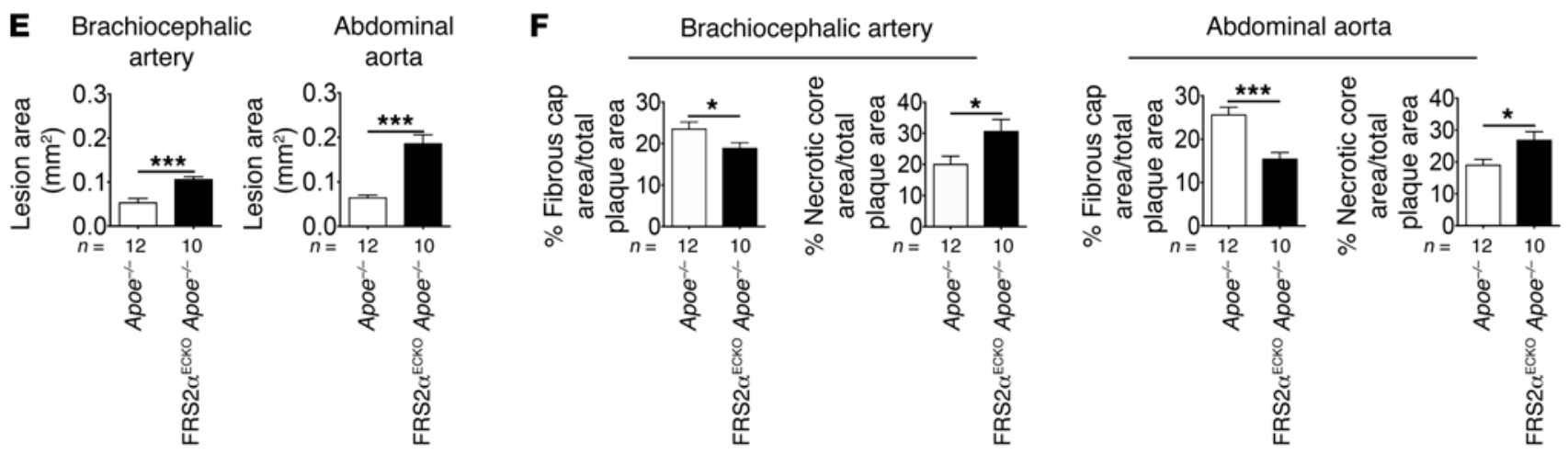

Figure 6. Effect of endothelial FGF signaling suppression on atherosclerosis in mice after 4 months of HFD. (A) Microphotographs of aortas from Apoe ${ }^{-1-}$ and FRS2 $\alpha^{\mathrm{ECKO}} \mathrm{Apoe}^{-/-}$mice ( 24 weeks of age) after 4 months of HFD in the en face preparation after staining with Oil Red 0 and quantification of lesion areas (10 mice per group). All data are shown as mean $\pm S D\left({ }^{* *} P<0.001\right.$ compared with $A p o e^{-1-}$; unpaired 2-tailed Student's $t$ test). (B) Representative examples of cross sections from the aortic root stained with Oil Red 0 and quantification of aortic root lesion areas (12 mice per group). Scale bar: $200 \mu \mathrm{m}$. Mean \pm SD ( ${ }^{* *} P<0.01$ compared with $A p o e^{-1-}$; unpaired 2-tailed Student's $t$ test). (C and D) Representative images of Oil Red 0- or Movat-stained brachio-

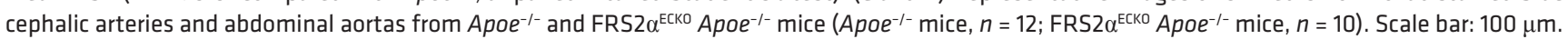
High-magnification images of the lesion shown in boxed areas are shown to the right. Scale bar: $50 \mu \mathrm{m}$. NC, necrotic core. Note circumferential plaque in FRS2 $\alpha^{\mathrm{ECKO}} \mathrm{Apoe}^{-1-}$ mice. (E) Measurement of lesion area ( ${ }^{* * *} P<0.001$ compared with Apo ${ }^{-1-}$; unpaired 2-tailed Student's $t$ test). (F) Quantifications of the extent of fibrous cap and necrotic areas in brachiocephalic arteries and abdominal aortas of Apoe ${ }^{-/-}$and $\mathrm{FRS} 2 \alpha^{\mathrm{ECKO}} A p o e^{-/-}$mice $\left({ }^{*} P<0.05\right.$; ${ }^{* * *} P<0.001$ compared with $\mathrm{Apoe}^{-1-}$; unpaired 2-tailed Student's $t$ test).

plaque growth by increasing deposition of fibronectin, a known proatherogenic ECM protein $(17,18)$, and increasing endothelial expression of adhesion molecules, such as ICAM and VCAM, thereby further promoting recruitment of circulating monocytes and leukocytes and inducing formation of new mesenchymal cells, leading to expansion of neointima.
A number of observations support this hypothesis. First, ECs downregulated FGFR1 expression and activated TGF- $\beta$ signaling when exposed to OSS or certain inflammatory mediators. Second, mice with endothelial-specific disruption of FGF signaling input developed EndMT and exhibited early and extensive development of atherosclerosis when crossed onto the Apoe ${ }^{-/-}$back- 
A

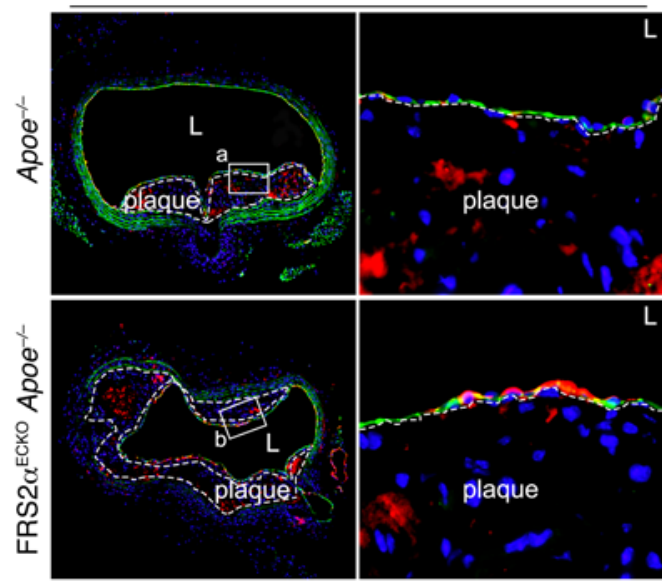

C

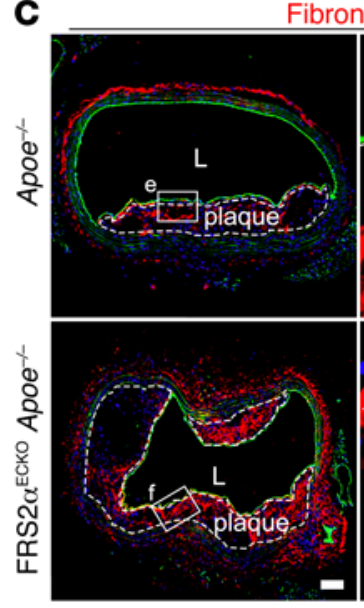

ICAM-1

ibronectin

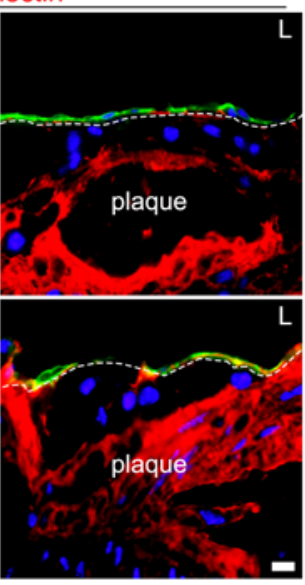

Cdh5-GFP/DAP

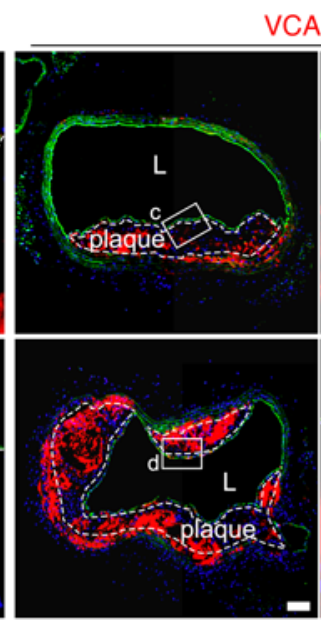

VCAM-1

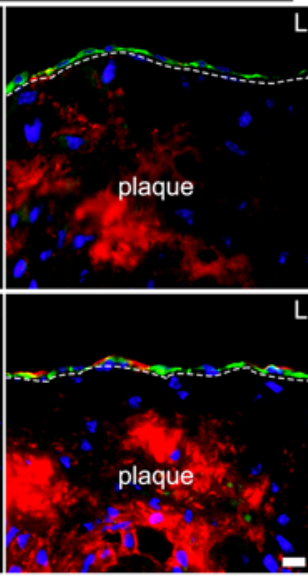

Cdh5-GFP/DAPI
B

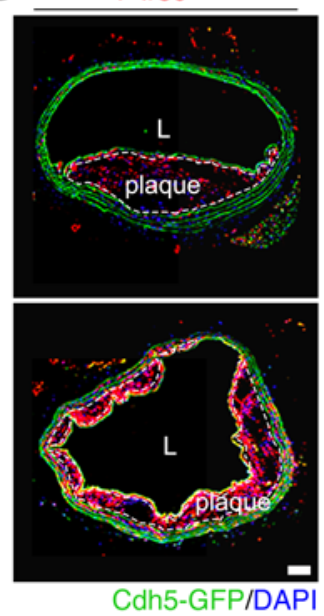

D

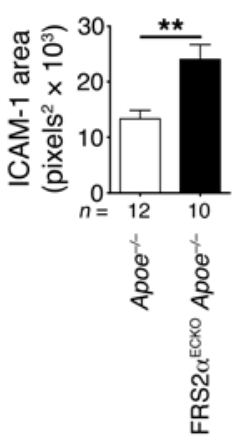

$\mathbf{E}$

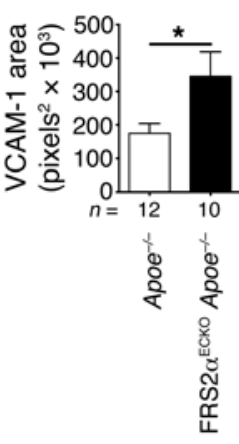

$\mathbf{F}$

Figure 7. Effect of endothelial FGF signaling suppression on inflammation and EndMT marker gene expression in mice. (A-C) Histological analysis of atherosclerotic plaques with anti-ICAM-1, anti-VCAM-1, anti-F4/80, and anti-fibronectin antibodies (Apoe $e^{-/-}$mice, $n=12 ; \mathrm{FRS2} \alpha^{\mathrm{ECKO}} \mathrm{Apoe}^{-/-}$mice, $n=10$ ). Note circumferential plaque in FRS2 $\alpha^{\text {ЕСКO }} \mathrm{Apoe}^{-/-}$mice. Nuclei were counterstained with DAPI (blue). Scale bar: $62 \mu \mathrm{m}$ (low-magnification images); $10 \mu \mathrm{m}$ (high-magnification images). High-magnification images of the boxed areas are shown to the right. (D-F) Measurement of F4/80, ICAM-1, VCAM-1, and fibronectin area $\left({ }^{*} P<0.05 ;{ }^{* *} P<0.01 ;{ }^{* *} P<0.001\right.$ compared with $A p o e^{-1-}$; unpaired 2-tailed Student's $t$ test).

ground. Third, analysis of human left main coronary arteries from patients with various extents of atherosclerosis demonstrated a strong inverse correlation between the extent of coronary endothelial FGFR1 expression, the appearance of p-SMAD2 signal, and EndMT and the severity of CAD in patients. Taken together, these results establish a link between inflammation, shear stress, the loss of protective endothelial FGF signaling input, and the induction of EndMT that, in turn, plays an important role in development and growth of atherosclerotic lesions.

The strong correlation we observed between the decline in endothelial FGFR1 expression and the extent of EndMT in atherosclerotic lesions is in agreement with the previous demonstration of a protective role of endothelial FGF signaling $(10,14)$. The two key factors regulating endothelial FGFR1 expression, disturbed shear stress and vessel wall inflammation, are also well-known atherosclerosis risk factors $(1,5)$. However, the link between disturbed shear stress and endothelial FGFR1 expression has not previously been reported. In this study, we show that exposure of cul- tured ECs in vitro to OSS, but not atheroprotective LSS, results in a decrease in FGFR1 expression and an increase in p-SMAD2. Interestingly, in vivo, there were no differences in endothelial FGFR1 levels in atheroprotected and atheroprone endothelium in mice on normal chow diet. However, HFD induced a much more significant reduction in FGFR1 levels in the atheroprone endothelium.

Inflammation is an important part of atherogenesis. Studies over the last decade have documented the presence of monocytederived macrophages, T cells, B cells, and other immune mediators in atherosclerotic lesions (19-21). This inflammatory milieu is thought to be due to a combination of factors, including mechanical factors, such disturbed shear stress (2) and lipid-induced changes in the vascular wall. With regard to the former, a number of studies have linked disturbed shear stress and endothelial production of fibronectin $(17,22)$, a well-established marker of epithelial-to-mesenchymal transition $(23,24)$. Fibronectin has a major role in promoting inflammatory signaling in ECs $(2,21)$, and multiple studies in mice demonstrate a causal role for fibronectin 
A

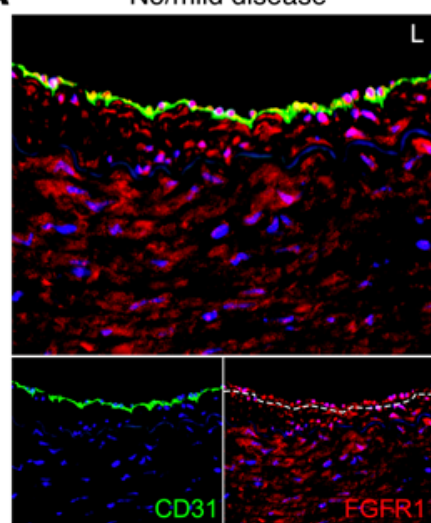

C

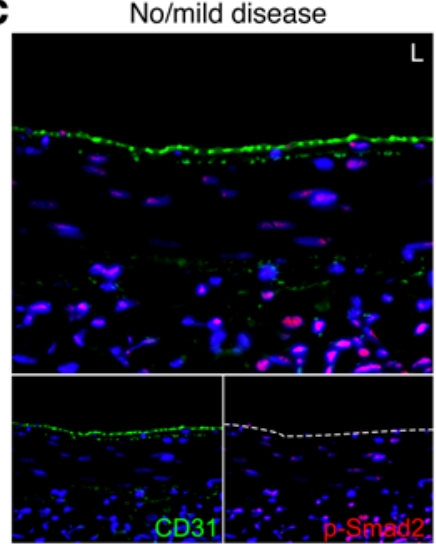

Moderate disease

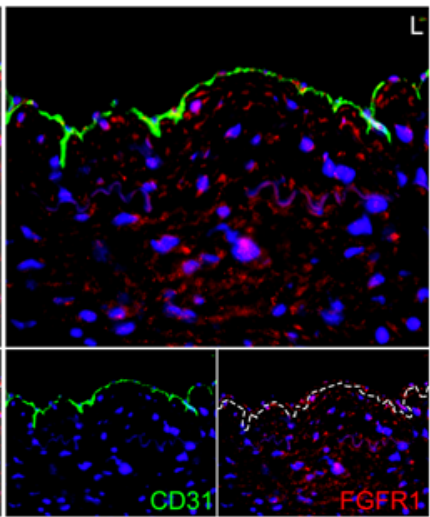

Moderate disease

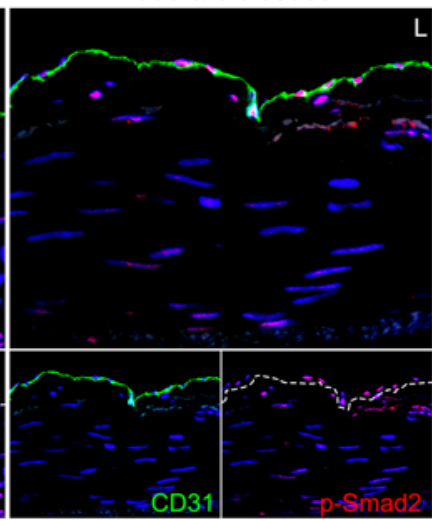

Severe disease

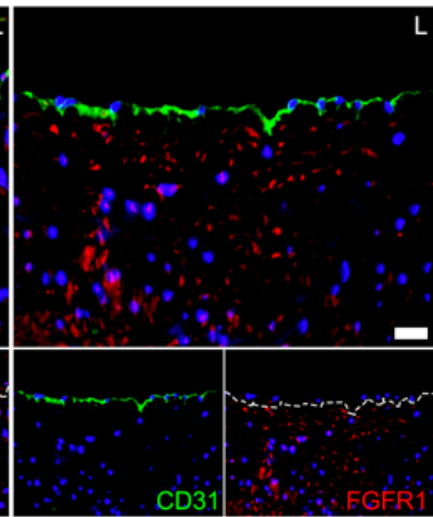

CD31/FGFR1/DAPI

Severe disease

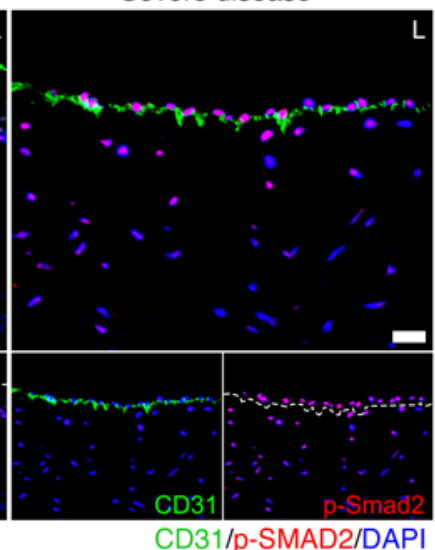

B

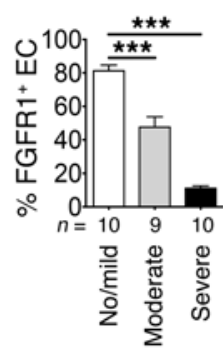

D

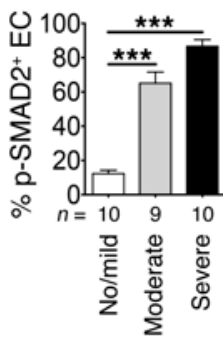

E

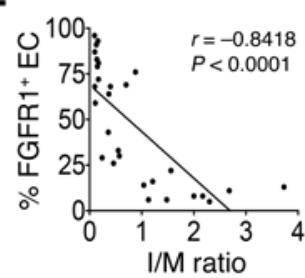

F

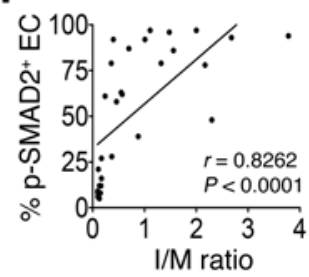

G

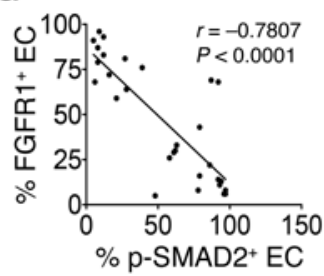

Figure 8. FGFR1 expression and SMAD2 phosphorylation in ECs in human coronary arteries. Immunocytochemical analysis of (A) FGFR1 and (C) p-SMAD2 expression in the endothelium of left main coronary arteries of patients with no/mild $(n=10)$, moderate $(n=9)$, and severe $(n=10)$ CAD. Representative images of immunofluorescence staining for CD31 (green) and FGFR1 (red) or p-SMAD2 (red). Nuclei were stained with DAPI (blue). Scale bar: $16 \mu \mathrm{m}$. (B and

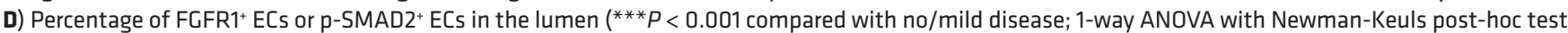
for multiple comparison correction). (E and F) Scatter plots of FGFR1 or p-SMAD2 and the I/M ratio. The corresponding Spearman's correlation coefficient ( $r$ ) between FGFR1 or p-SMAD2 and the I/M ratio and the $P$ value are shown. (C) Scatterplot of FGFR1 and p-SMAD2. The corresponding Spearman's correlation coefficient $(r)$ between FGFR1 and p-SMAD2 and the $P$ value are shown.

in promoting atherosclerosis $(18,25)$. Fibronectin production as a consequence of EndMT therefore appears to be one mechanism by which this process promotes inflammation and atherosclerosis.

At the same time, cholesterol crystal formation in lipid-loaded macrophages in the forming atheroma can lead to activation of the inflammasome, thereby promoting conversion of pro-IL-1 $\beta$ into an active cytokine (26). The preponderance of the M1 subset of macrophages in the atherosclerotic lesions (27) provides further evidence of the presence of inflammation, as these macrophages are thought be derived from monocytes in response to stimulation with IFN- $\gamma$. The latter is likely produced by $\mathrm{T}$ cells that in human lesions exhibit a Th1 secretion profile that includes IFN- $\gamma$ and
TNF- $\alpha$ (4). The pathogenic role of IFN- $\gamma$ in atherosclerosis is further demonstrated by its ability to induce lesion progression (28, 29), while its deficiency leads to a marked reduction in lesion size while not affecting the overall lipid profile (30). Similarly, activation of natural killer cells, also found in human atheroma lesions (19), results in secretion of IFN- $\gamma$ and TNF- $\alpha$. Thus, the atheroma is rich in inflammatory mediators and exhibits evidence of chronic activation of various inflammatory pathways.

While the link between inflammation and atherosclerosis is well established, the molecular underpinning of this connection remains poorly described. We propose that EndMT provides one such potential link. The ongoing inflammation, as well as dis- 

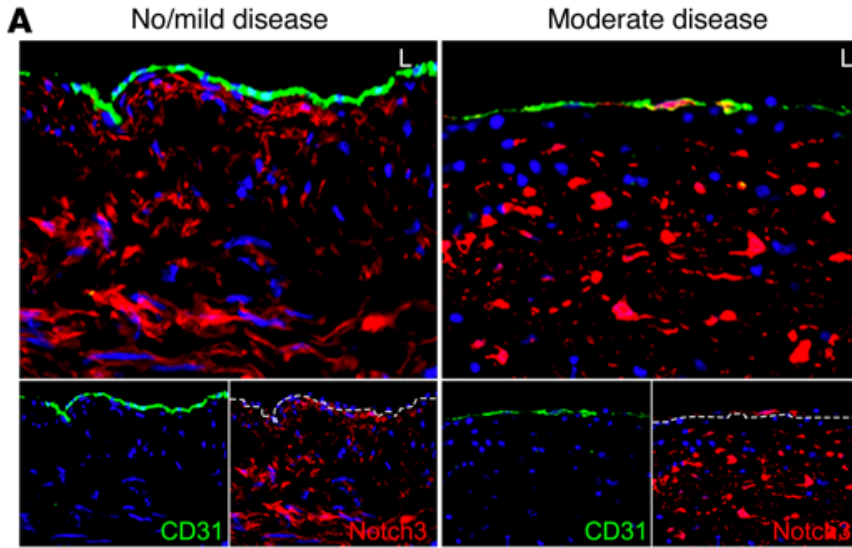

C
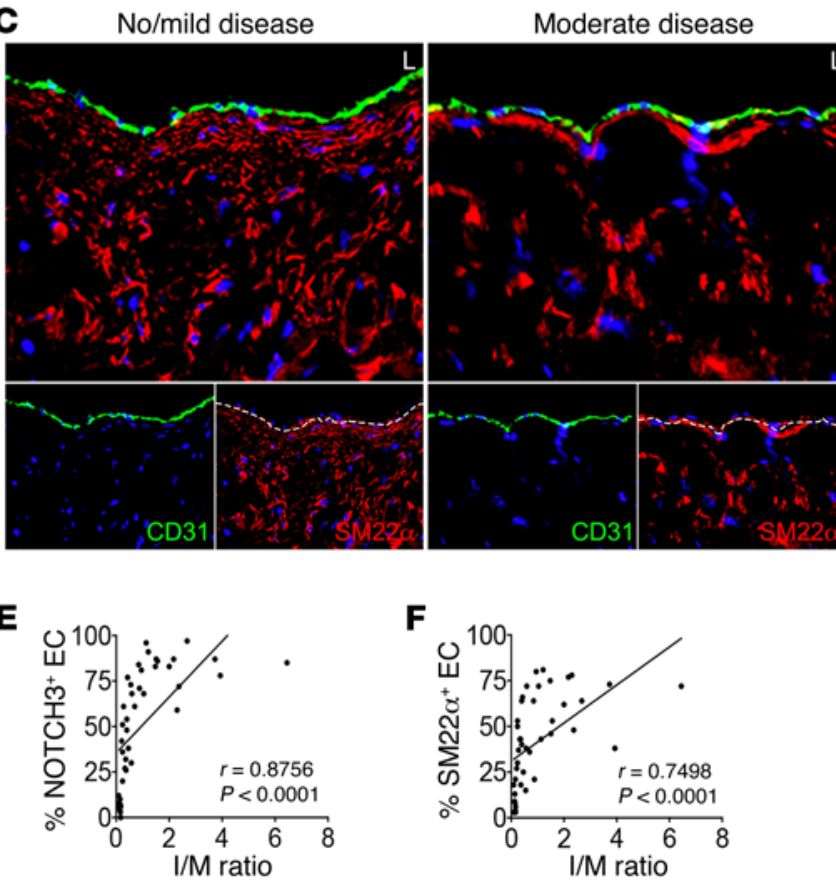

$\mathbf{F}$

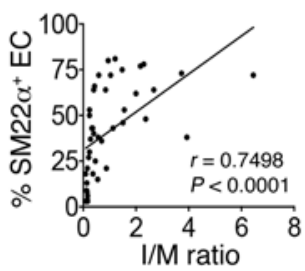

B
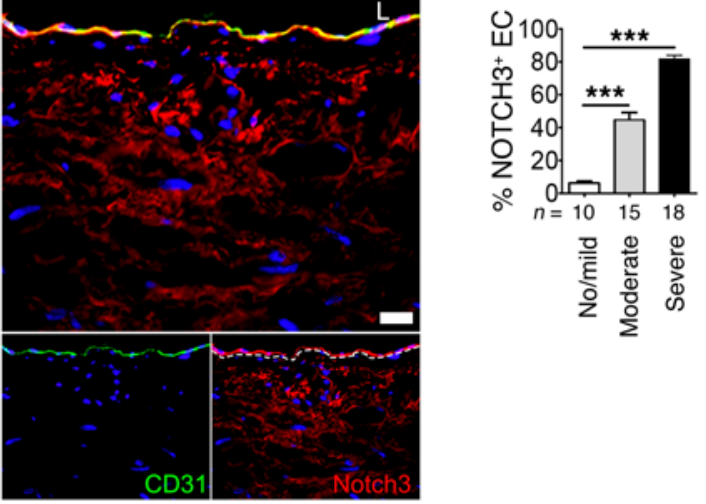

CD31/NOTCH3/DAPI

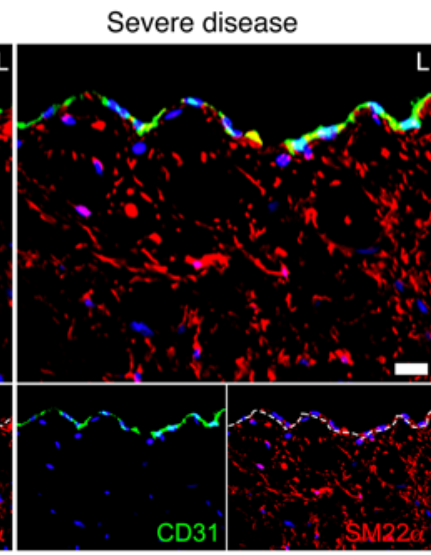

CD31/SM22 $\alpha / \mathrm{DAP}$
D

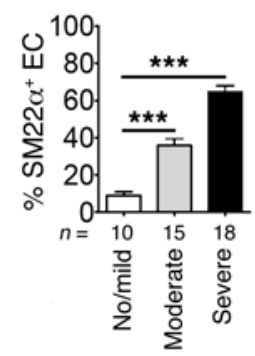

Figure 9. Smooth muscle marker expression in the endothelium of human coronary arteries. Left main coronary arteries from patients with no/mild $(n=10)$, moderate $(n=15)$, and severe $(n=18)$ CAD were evaluated. (A and $\mathbf{C})$ Representative images of immunofluorescence staining of left main coronary arteries for CD31 (green), NOTCH3 (red), and SM22 $\alpha$ (red). Nuclei were stained with DAPI (blue). Scale bar: $16 \mu \mathrm{m}$. (B and D) Percentage of NOTCH3 ${ }^{+}$ECs or SM22 $\alpha^{+}$ECs in the lumen ${ }^{* * *} P<0.001$ compared with no/mild disease; 1 -way ANOVA with Newman-Keuls post-hoc test for multiple comparison correction). (E and F) Scatter plots of $\mathrm{NOTCH}^{+}$ECs or SM22 $\alpha^{+}$ECs in the lumen and the I/M ratio. The corresponding Spearman's correlation coefficient ( $r$ ) between $\mathrm{NOTCH}^{+}$ECs or SM22 $\alpha^{+}$luminal ECs and the I/M ratio and the $P$ value are shown.

turbed shear stress, decrease endothelial FGFR1 expression, leading to activation of TGF- $\beta$ signaling, thereby inducing EndMT. The latter is responsible for a number of proatherogenic consequences, including increased expression of adhesion molecules, such as ICAM-1 and VCAM-1, thus promoting a further influx of inflammatory cells; deposition of extracellular matrix, such as fibronectin, that potentiates inflammation, thus accelerating atheroma growth; and, finally, the appearance of new mesenchymal cells that give rise to SMCs and fibroblasts in the neointima, further promoting plaque expansion. The proinflammatory phenotype of ECs undergoing EndMT establishes a feed-forward loop that further aggravates inflammation and leads to the ongoing plaque growth. Suppression of this process may be the key step in halting the progression of atherosclerosis.
Reduction of inflammation has recently become the key target in the development of new antiatherosclerotic therapies. Various attempts to suppress specific aspects of inflammation, including an IL-1 $\beta$-neutralizing antibody (CANTOS) (31), have been equivocal at best. Yet, the emerging biology of EndMT suggests that approaches targeting individual inflammatory pathways are likely to fail, since any of the "big 3" cytokines found in the plaque - IFN- $\gamma$, IL-1 $1 \beta$, and TNF- $\alpha$ - can induce EndMT and, hence, lead to atherosclerotic disease progression. In contrast, restoration of effective endothelial FGF signaling and/or suppression of endothelial TGF- $\beta$ signaling may effectively halt the disease progression. The latter points to the complexity of endothelial biology, as smooth muscle TGF- $\beta$ signaling has been linked to reduction in atherogenesis $(32,33)$ and may explain the ambiguous nature of TGF- $\beta$ effects in atherosclerosis (34). 
A

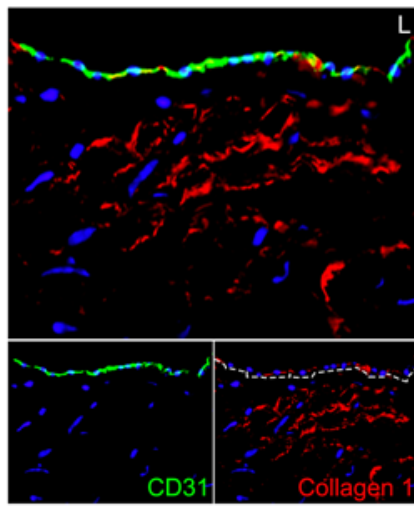

c

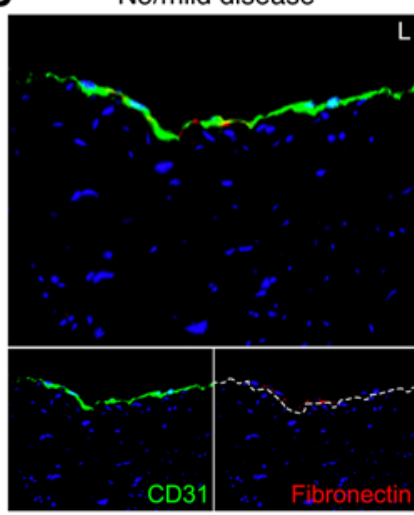

E

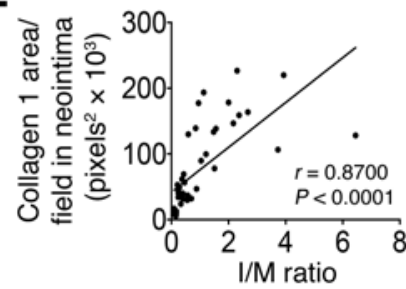

Moderate disease

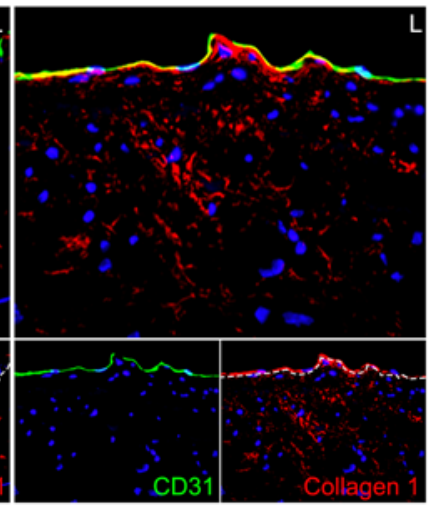

Moderate disease

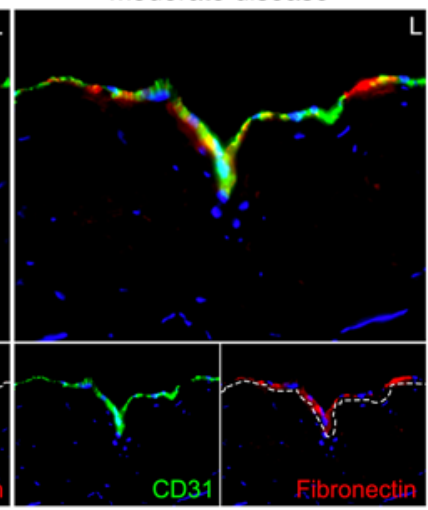

$\mathbf{F}$

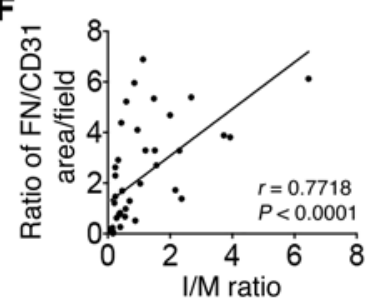

B

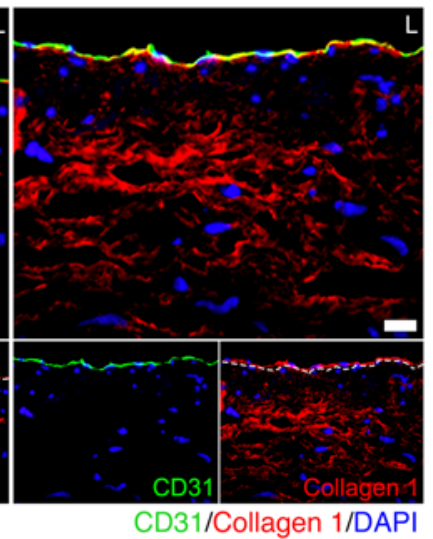

Severe disease

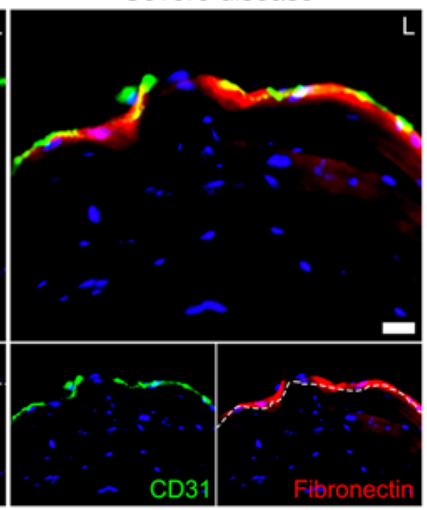

CD31/Fibronectin/DAPI

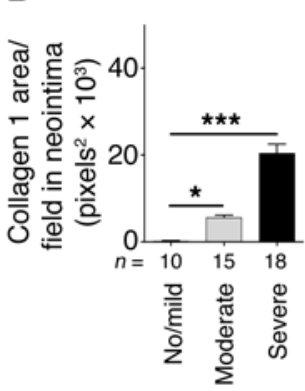

D

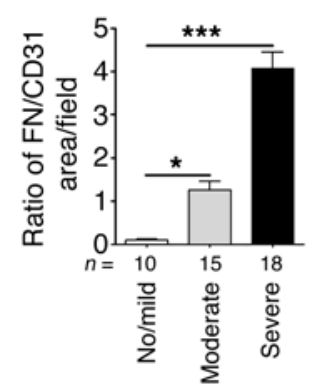

Figure 10. Mesenchymal marker expression in the endothelium of human coronary arteries. Left main coronary arteries from patients with no/mild $(n=10)$, moderate $(n=15)$, and severe $(n=18)$ CAD were evaluated. (A and $\mathbf{C})$ Representative images of immunofluorescence staining of left main coronary arteries for CD31 (green), collagen 1 (red), and fibronectin (red) in patients. Nuclei were stained with DAPI (blue). Scale bar: $16 \mu \mathrm{m}$. (B) Measurement of the collagen 1 area in the neointima per field $\left({ }^{*} P<0.05\right.$ compared moderate disease to no/mild disease; ${ }^{* *} P<0.001$ compared severe disease to no/mild dis ease; 1-way ANOVA with Newman-Keuls post-hoc test for multiple comparison correction). (D) Ratio of fibronectin area to CD31 area per field in the lumen $\left({ }^{*} P<0.05\right.$ compared moderate disease to no/mild disease; ${ }^{* *} P<0.001$ compared severe disease to no/mild disease; 1 -way ANOVA with Newman-Keuls post-hoc test for multiple comparison correction). (E and $\mathbf{F}$ ) Scatter plots of collagen 1 area in the neointima per field and the ratio of fibronectin area to CD31 area per field in the lumen and the I/M ratio. The corresponding Spearman's correlation coefficient ( $r$ ) between collagen 1 area in the neointima per field and the ratio of fibronectin area to CD31 area per field and the I/M ratio and the $P$ value are shown.

In summary, EndMT, induced by inflammatory components of the atheroma, potentiates the inflammatory milieu of atherosclerotic lesions and leads to progressive growth of the atherosclerotic plaque. Interventions aimed at controlling this process will test its role in progression of atherosclerosis and may, if successful, offer effective new approaches to reversing the natural history of this disease.

\section{Methods}

Cell culture and reagents. HUVECs (passage 5-10; CC-2517, Lonza) were cultured in endothelial basal medium-2, supplemented with the EGM-2MV BulletKit (CC-3202, Lonza), or in M199 with ECGS, supplemented with 20\% FBS (Sigma-Aldrich), L-glutamine (Gibco), and penicillin/streptomycin (Gibco). Human coronary aortic SMCs (HCASMCs) (C-017-5C), media (M231-500), and supplements (SMGS, S-007-25; SMDS, S-008-5) were purchased from Life Technologies. The cells were grown at $37^{\circ} \mathrm{C}, 5 \% \mathrm{CO}_{2}$, in Medium 231 (Life Technologies), supplemented with smooth muscle growth supplement (SMGS containing 4.9\% FBS, $2 \mathrm{ng} / \mathrm{ml} \mathrm{FGF2,} 0.5 \mathrm{ng} / \mathrm{ml} \mathrm{EGF,} 5 \mathrm{ng} / \mathrm{ml}$

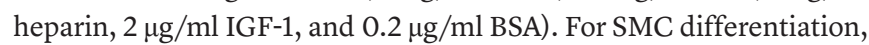
HCASMCs were incubated with Medium 231 containing smooth muscle differentiation supplement (SMDS containing 1\% FBS and $30 \mathrm{~g} / \mathrm{ml}$ heparin) for 8 days. For different cytokine treatment, HUVECs or 
A
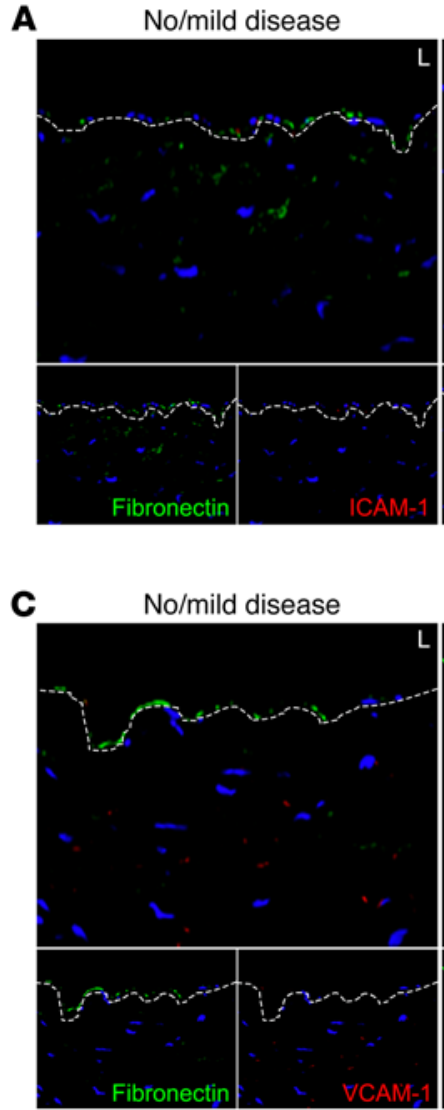

E

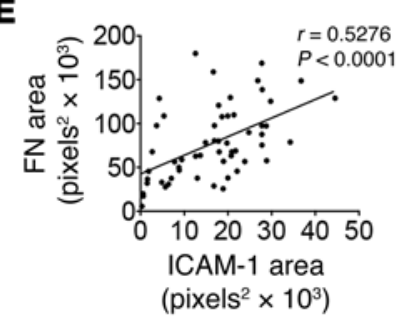

Moderate disease

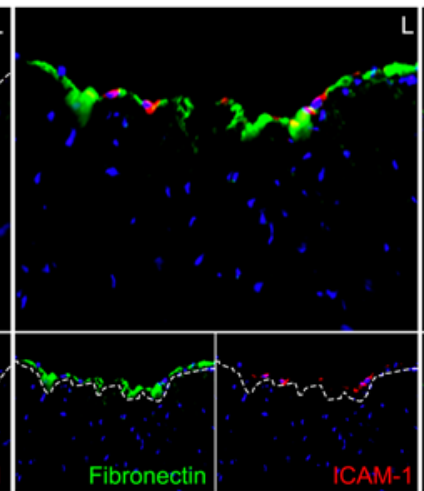

Moderate disease

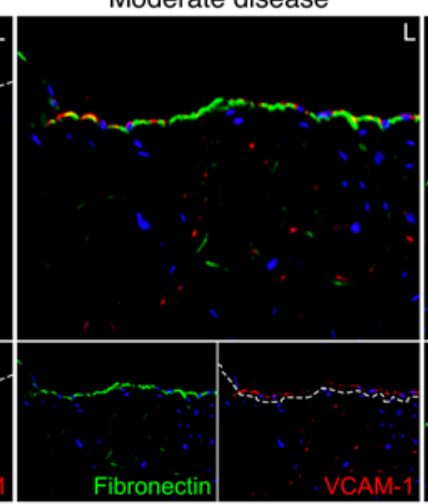

F

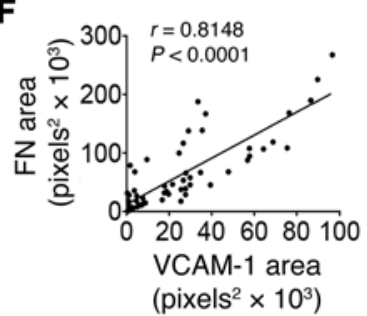

B

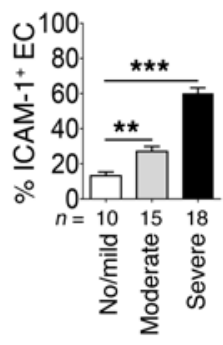

D

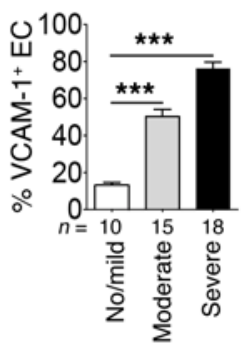

Figure 11. Mesenchymal and inflammatory marker expression in the endothelium of human coronary arteries. Left main coronary arteries from patients with no/mild $(n=10)$, moderate $(n=15)$, and severe $(n=18)$ CAD were evaluated. (A and $\mathbf{C})$ Representative images of immunofluorescence staining of left main coronary arteries for fibronectin (green), ICAM-1 (red), and VCAM-1 (red) in patients. Nuclei were stained with DAPI (blue). Scale bar: $16 \mu \mathrm{m}$. (B) Percentage of ICAM-1 $1^{+}$ECs in the lumen ${ }^{* *} P<0.01$ compared moderate disease to no/mild disease; ${ }^{* * *} P<0.001$ compared moderate disease to no/mild disease; ${ }^{* *} P<0.001$ compared severe disease to no/mild disease). (D) Percentage of VCAM-1+ ECs in the lumen (*** $P<0.001$ compared moderate disease to no/mild disease; ${ }^{* *} P<0.001$ compared severe disease to no/mild disease; 1-way ANOVA with Newman-Keuls post-hoc test for multiple comparison correction). (E and F) Scatter plots of fibronectin area and ICAM-1 and VCAM-1 area per field in the lumen. The corresponding Spearman's correlation coefficient $(r)$ between fibronectin area and ICAM-1 and VCAM- 1 area per field and the $P$ value are shown.

HCASMCs were treated with human IFN- $\gamma$ (300-02, PeproTech), human TNF- $\alpha$ (300-01A, Peprotech), or human IL-1 $\beta$ (200-01B, Peprotech) in complete growth medium or in differentiation medium.

Fluid shear stress. HUVECs were seeded on tissue culture plastic slides coated with $10 \mu \mathrm{g} / \mathrm{ml}$ fibronectin and grown to confluence in M199 culture media supplemented with 20\% FBS, ECGS, 100 mg/l heparin, and penicillin/streptomycin. Cells were starved in M199 media supplemented with $8 \%$ FBS 4 hours prior stimulation. Slides were then clamped into a parallel plate flow chamber and sheared at 12 dynes $/ \mathrm{cm}^{2}$ (laminar flow) or $1 \pm 4$ dynes $/ \mathrm{cm}^{2}$ (oscillatory flow) for 16 or 48 hours. Cells were then washed in PBS and further processed for immunostaining or mRNA extraction.
SMAD2/3 translocation assay. Cells were fixed with $4 \%$ formaldehyde for 15 minutes and then permeabilized with $1 \%$ Triton X-100 for 15 minutes. After permeabilization, cells were blocked with StartingBlock Blocking Buffer (Thermo Fisher Scientific) for 15 minutes and incubated overnight at $4^{\circ} \mathrm{C}$ with a SMAD2/3 primary antibody diluted in StartingBlock Blocking Buffer (1:500, Cell Signaling). After 3 washing steps, cells were probed with DAPI and Alexa Fluor 647-conjugated secondary antibody (1:500, Molecular Probes) diluted in StartingBlock Blocking Buffer for 2 hours at room temperature. Cells were then mounted with Fluoromount-G Mounting Media (Southern Biotech). Fifty images were then captured with a $\times 20$ objective mounted on a Perkin Elmer spinning disk confocal microscope equipped with 
a motorized stage (Prior Scientific). Images were then analyzed with a proprietary MATLAB function as described previously (35). Briefly, masks of the images were made using a combination of an adaptive histogram equalization algorithm with intensity and size thresholding. Cell orientation was calculated by taking the masks of the cell nuclei (determined from DAPI images), fitting them to an ellipse, and determining the angle between the flow direction and the major axis of the ellipse. Nuclei eccentricity was measured on the basis of the eccentricity of the fitted ellipse. Nuclear translocation was computed by taking the mask of the transcription factor stain (SMAD2/3), calculating the product of both the area and the intensity of the stain present in the nucleus, and dividing it by the product of the total stain area and the total intensity of the stain. This yields a unitless metric we termed the translocation factor, which equals 1 in cells with complete nuclear translocation and $\mathrm{O}$ in cells with no translocation.

Generation of lentiviruses. The human FGFR1 shRNA lentiviral construct was purchased from Sigma-Aldrich and the human FRS2 $\alpha$ shRNA lentiviral construct was purchased from Open Biosystems. For the production of shRNA lentivirus, $3.7 \mu \mathrm{g} \Delta 8.2,0.2 \mu \mathrm{g}$ VSVG, and $2.1 \mu \mathrm{g}$ pLKO. 1 carrying the control, FGFR1, and FRS $2 \alpha$ shRNA were cotransfected into 293T cells using X-tremeGENE 9 DNA Transfection Reagent (06365787001, Roche). Forty-eight hours later, the medium was harvested, cleared by a $0.45-\mu \mathrm{m}$ filter (PN4184, PALL Life Sciences), mixed with polybrene (5 $\mathrm{g} / \mathrm{ml})$ (H9268, Sigma-Aldrich), and applied to the cells. After 6 hours of incubation, the virus-containing medium was replaced by fresh medium. Virus supernatants were tittered using the QuickTiter Lentivirus Titer Kit (Lentivirus-Associated HIV p24, VPK-107, Cell Biolabs).

RNA isolation and quantitative real-time PCR. Cells were suspended in TRIzol Reagent (15596018, Invitrogen), and total RNA (74134, QIAGEN) was isolated according to the manufacturer's instructions. Reverse transcriptions were performed by using the iScript cDNA Synthesis Kit (170-8891, Bio-Rad). Quantitative real-time PCR (qRT-PCR) was performed using the Bio-Rad CFX94 by mixing equal amount of cDNAs, iQ SYBR Green Supermix (170-8882, Bio-Rad), and gene-specific primers from SABiosciences (a QIAGEN company) (FGFR1 [PPH00372F], VE-cadherin [PPH00668F], N-cadherin [PPHO0636F], eNOS [PPH01298F], TGF-ßR1 [PPH00237C], PAI-1 [PPHO0215F], ICAM-1 [PPH00640F], VCAM-1 [PPH00623E], MCP-1 [PPH00192F], ZEB2 [PPH09021B], Slug [PPH02475A], and Snail [PPH02459A]) or the following primers for detecting mRNA: TWIST1, actggcctgcaaaaccatag and tgcattttaccatgggtcct; ACTA2, gccacagcagcttcctcttc and tgaattccagcggactccat; $\mathrm{NOTCH}$, ctcatccgaaaccgetctac and tcttccaccatgccetctac; fibronectin 1, gttgtgtccggcttgactcc and agtgtcagggtttgcctcca; and collagen $1 \mathrm{~A}$, tgtggcccagaagaactggt and caggaaggtcagctggatgg. All reactions were done in a $25 \mu \mathrm{l}$ reaction volume in duplicate. Individual mRNA expression was normalized in relation to expression of endogenous $\beta$-actin. PCR amplification consisted of 10 minutes of an initial denaturation step at $95^{\circ} \mathrm{C}$, followed by 46 cycles of PCR at $95^{\circ} \mathrm{C}$ for 15 seconds and $60^{\circ} \mathrm{C}$ for 30 seconds.

Antibodies used for immunodetection of proteins. We used the following antibodies for immunoblotting (IB), immunofluorescence (IF), or immunohistochemistry (IHC): CD31 (sc-1506, Santa Cruz; IHC for human and mouse paraffin samples), CD31 (sc-8306, Santa Cruz; IB), CD31 (M0823, Dako; IHC for human frozen samples), collagen 1 (600-401-103S, Rockland; IB), collagen 1 (NB600-408, Novus Biologicals; IHC), p44/42 MAPK (ERK1/2) (Thr202/Tyr204) (4370, Cell
Signaling; IHC), F4/80 (ab6640, abcam; IHC), fibronectin (F0916, Sigma-Aldrich; IB), fibronectin (F3648, Sigma-Aldrich; IHC), FGFR1 (2144-1, Epitomics; IB), FGFR1 (ab10646, abcam; IHC for mouse and human paraffin samples), FGFR1 (FB817; IHC for human paraffin samples) (14), FRS2 $\alpha$ (ab10425, abcam; IHC), FRS2 (sc-8318, Santa Cruz; IB), GAPDH (2118, Cell Signaling; IB), HSP90 (SAB4300541, SigmaAldrich; IB), ICAM-1 (MCA532, AbD Serotec; IHC for human tissue), ICAM-1 (116102, BioLegend; IHC for mouse tissue), ICAM-1 (3482-1, Epitomics; IB), N-cadherin (610920, BD; IB), eNOS (610297, BD; IB), NOTCH3 (5276, Cell Signaling; IB), NOTCH3 (ab23426, abcam; IHC), PAI-1 (612024, BD; IB), SM22 $\alpha$ (ab14106, abcam; IB, IHC), smooth muscle-MHC (M7786, Sigma-Aldrich; IB), p-SMAD2 (Ser ${ }^{465 / 467}$ ) (3101, Cell Signaling; IHC for human paraffin samples), p-SMAD2 (Ser ${ }^{465 / 467}$ ) (AB3849, Millipore; IHC for mouse paraffin samples), p-SMAD2 $\left(\right.$ Ser $\left.^{465 / 467}\right)$ (3108, Cell Signaling; IB), SMAD2 (3122, Cell Signaling; IB), SMAD2/3 (8685, Cell Signaling; IF), smooth muscle $\alpha$-actin (A2547, Sigma-Aldrich; IB), smooth muscle $\alpha$-actin-allophycocyanin (IC1420A, R\&D Systems; IHC), TGF- $\beta$ R1 (3712, Cell Signaling; IB), TGF- $\beta$ R1 (sc-398, Santa Cruz; IB), $\beta$-tubulin (T7816, Sigma-Aldrich; IB), VCAM-1 (NBP1-47491, Novus Biologicals; IHC for human tissue), VCAM-1 (ab19569, abcam; IHC for mouse tissue), VCAM-1 (3540-1, Epitomics; IB), VE-cadherin (sc-6458, Santa Cruz; IB), VEGFR2 (2479, Cell Signaling; IB), and vimentin (V6630, Sigma-Aldrich; IB).

Generation of mice. Frs $2 a^{f / f l}$ mice were previously described (36). Frs $2 a^{f / f l}$ mice were crossed to the Rosa26 fluorescent reporter $m T / m G$ reporter line (JAX SN:007676) and then crossed to C57BL/6 Apoe ${ }^{-/-}$ mice (JAXSN:002052). Frs $2 a^{f / f l} A p o e^{-/-} m T / m G$ offspring were bred with mice expressing Cre recombinase under the $C d h 5$ promoter (gift from R.H. Adams, Max Planck Institute, Münster, Germany). Apoe ${ }^{-/-}$and FRS $2 \alpha^{\mathrm{ECKO}}$ Apoe $^{-/-}$male mice were fed a Western diet (40\% kcal\% fat, $1.25 \%$ cholesterol, $0 \%$ cholic acid) for 4 or 16 weeks (product D12108, Research Diets), starting at the age of 8 weeks. Their body weight and blood lipid profile were indistinguishable from those of $A p o e^{-/-}$mice (Supplemental Figure 5, D and E). PCR genotyping analysis was done using the following primers: Frs $2 a^{f / / l}$ (5'-GAGTGTGCTGTGATTGGAAGGCAG-3' and 5'-GGCACGAGTGTCTGCAGACACATG-3'), $m T / m G$ (5'-CTCTGCTGCCTCCTGGCTTCT-3', 5'-CGAGGCGGATCACAAGCAATA-3', and 5'-TCAATGGGCGGGGGTCGTT-3'), Cdh5-CreER ${ }^{T 2}$ (5'-GCCTGCATTACCGGTCGATGCAACGA-3' and 5'-GTGGCAGATGGCGCGGCAACACCATT-3'), Apoe (5'-GCCTAGCCGAGGGAGAGCCG-3', 5'-GTGACTTGGGAGCTCTGCAGC-3', and 5'-GCCGCCCCGACTGCATCT-3').

Immunohistochemical staining. Blocks were sectioned at $5-\mu \mathrm{m}$ intervals using a Microm cryostat (for frozen blocks) or a Paraffin Microtome (for paraffin blocks). For frozen tissue sections, slides were fixed in acetone for 10 minutes at $-20^{\circ} \mathrm{C}$. For paraffin sections, slides were dewaxed in xylene, boiled for 20 minutes in citrate buffer (10 mM, pH 6.0) for antigen retrieval, and rehydrated. After washing 3 times with PBS, tissue sections were incubated with primary antibodies diluted in blocking solution (10\% BSA and horse serum in PBS) overnight at $4^{\circ} \mathrm{C}$ in a humidified chamber. Sections were washed 3 times with Tris-buffered saline; incubated with appropriate Alexa Fluor 488-, Alexa Fluor 594-, or Alexa Fluor 647-conjugated secondary antibodies diluted 1:1,000 in blocking solution for 1 hour at room temperature; washed again 3 times, and mounted on slides with ProLong Gold Mounting Reagent with DAPI (P36935, Life Technologies). All immunofluorescence micrographs were acquired using a Axiovert 
200M microscopy system (Carl Zeiss MicroImaging). Images were captured using Velocity software, and quantifications were performed using ImageJ software (NIH).

Histological analysis of atherosclerotic lesions. The animals were euthanized and perfusion fixed with $4 \%$ paraformaldehyde (18814, Polysciences Inc.) via the left ventricle for 5 minutes. The lesions located in the aorta, aortic roots, brachiocephalic artery, and abdominal aorta were analyzed using Oil Red O staining. To measure lesions in the aorta, the whole aorta, including the ascending arch and thoracic and abdominal segments, was dissected, gently cleaned of adventitial tissue, and stained with Oil Red O (O0625, Sigma-Aldrich) as previously described (37). The surface lesion area was quantified with ImageJ software (NIH). To measure lesions in the aortic root, the heart and proximal aorta were excised, and the apex and lower half of the ventricles were removed.

Serum lipid analysis. Serum was obtained through centrifugation of the blood for 2 minutes at 9,391 $g$ at $4^{\circ} \mathrm{C}$ and stored at $-80^{\circ} \mathrm{C}$ until each assay was performed. Measurement of total cholesterol, triglyceride, and HDL cholesterol levels was performed in the Yale Mouse Metabolic Phenotyping Center.

Western blot analysis. Cells were lysed with HNTG lysis buffer (20 mM HEPES [pH 7.4], 150 mM NaCl, $10 \%$ glycerol, $1 \%$ Triton X-100, $1.5 \mathrm{mM} \mathrm{MgCl}$, $1.0 \mathrm{mM} \mathrm{EGTA)} \mathrm{containing} \mathrm{complete} \mathrm{Mini} \mathrm{EDTA-free}$ Protease Inhibitors (11836170001, Roche) and Phosphatase Inhibitors (04906837001, Roche). $20 \mu \mathrm{g}$ of total protein from each sample was resolved on Criterion TGX Precast Gels (567-1084, Bio-Rad) with Tris/Glycin/SDS Running Buffer (161-0772, Bio-Rad), transferred to nitrocellulose membranes (162-0094, Bio-Rad), and then probed with various antibodies. Chemiluminescence measurements were performed using SuperSignal West Pico Chemiluminescent Substrate (34080, Thermo Fisher Scientific).

Patient population. Human coronary arteries were obtained from the explanted hearts of transplant recipients or cadaver organ donors.

Specimen collection. Investigators were on call with the surgical team and collected the heart at the time of explant. To minimize ex vivo artifacts, an approximately 5- to 20-mm segment of the left main coronary artery was removed within the operating room (Supplemental Figure 8A) and immediately processed as frozen sections $(n=43)$ in Optimal Cutting Temperature Medium (Sakura Finetek USA Inc.), and, when of sufficient length, an additional segment ( $n=29)$ was also fixed in formalin for later embedding, sectioning, and staining. The majority of analyses were in frozen specimens, although detection by certain antibodies (FGFR1 and p-SMAD2) was more optimal in paraffin-embedded specimens.

Histology and morphometric analysis. Sections of left main coronary arteries were stained with H\&E and Movat. Digital H\&E- and Movat-stained photographs of one section from each block were pro- jected at final magnifications of $\times 40$. ImageJ software $(\mathrm{NIH})$ was used for morphometric analyses. As described in Supplemental Figure 8B, measurements were made of the intima and media thickness. The ratio of I/M thickness and plaque stages (38) were used to grade the severity of atherosclerosis. We averaged the results for these parameters from 4 different areas for each specimen to obtain mean values. Left main coronary arteries with an I/M ratio of less than 0.2 were considered as having no disease or mild disease; those with an I/M ratio of between 0.2 and 1 were considered as having moderate disease; those with an I/M ratio of greater than 1 or with calcification were considered as having severe disease.

Statistics. All graphs were created using GraphPad Prism software, and statistical analyses were calculated using GraphPad Prism. The significance of the differences between the controls and the experimental groups was determined using a 2-tailed Student's $t$ test. For multiple comparisons, 1-way ANOVA with Newman-Keuls test was used. Correlation analyses between variables were performed using the Pearson rank correlation test. A $P$ value of less than 0.05 was considered significant. All results were confirmed by at least 3 independent experiments. Error bars represent mean \pm SEM.

Study approval. All experiments involving animals were reviewed and approved by the Yale University Institutional Animal Care and Use Committee. The procedures related to human subjects were approved by the Institutional Review Boards of Yale University and the New England Organ Bank. A waiver for consent was approved for surgical patients, and written informed consent was obtained from a member of the family for deceased organ donors.

\section{Author contributions}

P-YC, LQ, GL, GT, MAS, and MS designed research; P-YC, LQ, NB, MB, TA, and GL performed research; P-YC, LQ, GL, GT, NB, and MS analyzed data; and P-YC and MS wrote the manuscript.

\section{Acknowledgments}

We thank Robert Friesel (Maine Medical Center Research Institute) for providing FGFR1 antibody and Ralf Adams (Max Planck Institute) for Cdh5-CreER ${ }^{T 2}$ mice. We are grateful to Rita Webber and Nicole Copeland for maintaining mouse colonies used in this study. This work was supported by NIH grants R01 HL053793 (to M. Simons) and P01 HL107205 (to M. Simons and M.A. Schwartz). The blood lipid profile was done by the Yale Mouse Metabolic Phenotyping Center core facility (NIH grant U24 DK059635).

Address correspondence to: Michael Simons, Yale University Internal Medicine, Section of Cardiovascular Medicine, PO Box 208017, 333 Cedar Street, New Haven, Connecticut 06520-8017, USA. Phone: 203.737.4643; E-mail:michael.simons@yale.edu.
1. Conway DE, Schwartz MA. Flow-dependent cellular mechanotransduction in atherosclerosis. J Cell Sci. 2013;126(pt 22):5101-5109.

2. Hahn C, Schwartz MA. Mechanotransduction in vascular physiology and atherogenesis. Nat Rev Mol Cell Biol. 2009;10(1):53-62.

3. Libby P, Ridker PM, Hansson GK. Progress and challenges in translating the biology of atherosclerosis. Nature. 2011;473(7347):317-325.

4. Libby P, Lichtman AH, Hansson GK. Immune effector mechanisms implicated in atherosclerosis: from mice to humans. Immunity. 2013;38(6):1092-1104.

5. Tabas I, Garcia-Cardena G, Owens GK. Recent insights into the cellular biology of atherosclerosis. J Cell Biol. 2015;209(1):13-22.

6. Zeisberg EM, et al. Endothelial-to-mesenchymal transition contributes to cardiac fibrosis. Nat Med. 2007;13(8):952-961.

7. Maddaluno L, et al. EndMT contributes to the onset and progression of cerebral cavernous malformations. Nature. 2013;498(7455):492-496.

8. Kitao A, et al. Endothelial to mesenchymal transition via transforming growth factor- $\beta 1 / \mathrm{Smad}$ activation is associated with portal venous stenosis in idiopathic portal hypertension. Am J Pathol. 2009;175(2):616-626.

9. Piera-Velazquez S, Li Z, Jimenez SA. Role of endothelial-mesenchymal transition (EndoMT) in the pathogenesis of fibrotic disorders. Am J 
Pathol. 2011;179(3):1074-1080.

10. Chen PY, et al. FGF regulates TGF- $\beta$ signaling and endothelial-to-mesenchymal transition via control of let-7 miRNA expression. Cell Rep. 2012;2(6):1684-1696.

11. Cooley BC, et al. TGF- $\beta$ signaling mediates endothelial-to-mesenchymal transition (EndMT) during vein graft remodeling. Sci Transl Med. 2014;6(227):227ra34.

12. Medici D, Shore EM, Lounev VY, Kaplan FS, Kalluri R, Olsen BR. Conversion of vascular endothelial cells into multipotent stem-like cells. Nat Med. 2010;16(12):1400-1406.

13. van Meeteren LA, ten Dijke P. Regulation of endothelial cell plasticity by TGF- $\beta$. Cell Tissue Res. 2012;347(1):177-186.

14. Chen PY, Qin L, Tellides G, Simons M. Fibroblast growth factor receptor 1 is a key inhibitor of TGF $\beta$ signaling in the endothelium. Sci Signal. 2014;7(344):ra90.

15. Ten Dijke P, Egorova AD, Goumans MJ, Poelmann RE, Hierck BP. TGF- $\beta$ signaling in endothelial-to-mesenchymal transition: the role of shear stress and primary cilia. Sci Signal. 2012;5(212):pt2.

16. Pitulescu ME, Schmidt I, Benedito R, Adams RH. Inducible gene targeting in the neonatal vasculature and analysis of retinal angiogenesis in mice. Nat Protoc. 2010;5(9):1518-1534.

17. Feaver RE, Gelfand BD, Wang C, Schwartz MA, Blackman BR. Atheroprone hemodynamics regulate fibronectin deposition to create positive feedback that sustains endothelial inflammation. Circ Res. 2010;106(11):1703-1711.

18. Rohwedder I, et al. Plasma fibronectin deficiency impedes atherosclerosis progression and fibrous cap formation. EMBO Mol Med. 2012;4(7):564-576.
19. Lahoute C, Herbin O, Mallat Z, Tedgui A. Adaptive immunity in atherosclerosis: mechanisms and future therapeutic targets. Nat Rev Cardiol. 2011;8(6):348-358.

20. Libby P. Inflammation in atherosclerosis. Arterioscler Thromb Vasc Biol. 2012;32(9):2045-2051.

21. Li X, Deroide N, Mallat Z. The role of the inflammasome in cardiovascular diseases. J Mol Med (Berl). 2014;92(4):307-319.

22. Gelfand BD, et al. Hemodynamic activation of $\beta$-catenin and T-cell-specific transcription factor signaling in vascular endothelium regulates fibronectin expression. Arterioscler Thromb Vasc Biol. 2011;31(7):1625-1633.

23. Jung HY, Fattet L, Yang J. Molecular pathways: linking tumor microenvironment to epithelialmesenchymal transition in metastasis. Clin Cancer Res. 2015;21(5):962-968.

24. Scanlon CS, Van Tubergen EA, Inglehart RC, D'Silva NJ. Biomarkers of epithelial-mesenchymal transition in squamous cell carcinoma. J Dent Res. 2013;92(2):114-121.

25. Tan MH, Sun Z, Opitz SL, Schmidt TE, Peters JH, George EL. Deletion of the alternatively spliced fibronectin EIIIA domain in mice reduces atherosclerosis. Blood. 2004;104(1):11-18.

26. Duewell P, et al. NLRP3 inflammasomes are required for atherogenesis and activated by cholesterol crystals. Nature. 2010;464(7293):1357-1361.

27. Stoger JL, et al. Distribution of macrophage polarization markers in human atherosclerosis. Atherosclerosis. 2012;225(2):461-468.

28. Gupta S, Pablo AM, Jiang X, Wang N, Tall AR, Schindler C. IFN- $\gamma$ potentiates atherosclerosis in ApoE knock-out mice. J Clin Invest. 1997;99(11):2752-2761.

29. Tellides G, et al. Interferon- $\gamma$ elicits arterio- sclerosis in the absence of leukocytes. Nature. 2000;403(6766):207-211.

30. Whitman SC, Ravisankar P, Daugherty A. IFN- $\gamma$ deficiency exerts gender-specific effects on atherogenesis in apolipoprotein $\mathrm{E}^{-/-}$mice. J Interferon Cytokine Res. 2002;22(6):661-670.

31. Everett BM, et al. Rationale and design of the Cardiovascular Inflammation Reduction Trial: a test of the inflammatory hypothesis of atherothrombosis. Am Heart J. 2013;166(2):199-207.e15.

32. Lutgens $\mathrm{E}$, et al. Transforming growth factor- $\beta$ mediates balance between inflammation and fibrosis during plaque progression. Arterioscler Thromb Vasc Biol. 2002;22(6):975-982.

33. Mallat $Z$, Tedgui A. The role of transforming growth factor $\beta$ in atherosclerosis: novel insights and future perspectives. Curr Opin Lipidol. 2002;13(5):523-529.

34. Toma I, McCaffrey TA. Transforming growth factor- $\beta$ and atherosclerosis: interwoven atherogenic and atheroprotective aspects. Cell Tissue Res. 2012;347(1):155-175.

35. Baeyens $\mathrm{N}$, et al. Syndecan 4 is required for endothelial alignment in flow and atheroprotective signaling. Proc Natl Acad Sci U S A. 2014;111(48):17308-17313.

36. Lin Y, Zhang J, Zhang Y, Wang F. Generation of an Frs $2 \alpha$ conditional null allele. Genesis. 2007;45(9):554-559.

37. Huang Q, et al. AIP1 suppresses atherosclerosis by limiting hyperlipidemia-induced inflammation and vascular endothelial dysfunction. Arterioscler Thromb Vasc Biol. 2013;33(4):795-804.

38. Virmani R, Kolodgie FD, Burke AP, Farb A, Schwartz SM. Lessons from sudden coronary death: a comprehensive morphological classification scheme for atherosclerotic lesions. Arterioscler Thromb Vasc Biol. 2000;20(5):1262-1275. 